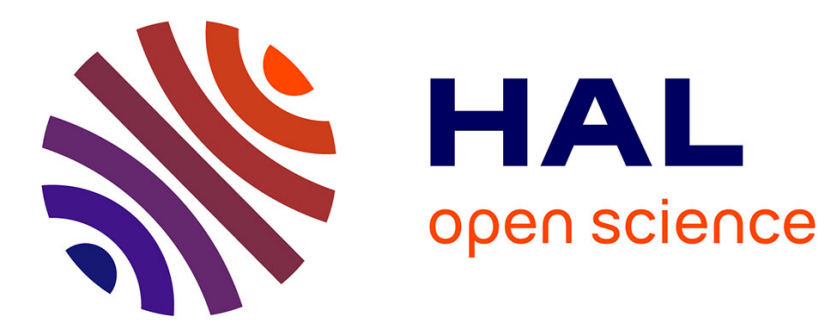

\title{
Effect of high frequency irradiation on the dynamical properties of weakly bound electrons
}

P. Avan, C. Cohen-Tannoudji, J. Dupont-Roc, C. Fabre

\section{To cite this version:}

P. Avan, C. Cohen-Tannoudji, J. Dupont-Roc, C. Fabre. Effect of high frequency irradiation on the dynamical properties of weakly bound electrons. Journal de Physique, 1976, 37 (9), pp.993-1009. 10.1051/jphys:01976003709099300 . jpa-00208505

\section{HAL Id: jpa-00208505 https://hal.science/jpa-00208505}

Submitted on 1 Jan 1976

HAL is a multi-disciplinary open access archive for the deposit and dissemination of scientific research documents, whether they are published or not. The documents may come from teaching and research institutions in France or abroad, or from public or private research centers.
L'archive ouverte pluridisciplinaire HAL, est destinée au dépôt et à la diffusion de documents scientifiques de niveau recherche, publiés ou non, émanant des établissements d'enseignement et de recherche français ou étrangers, des laboratoires publics ou privés. 


\title{
LE JOURNAL DE PHYSIQUE
}

Classification

Physics Abstracts

$2.600-3.340$

\section{EFFECT OF HIGH FREQUENCY IRRADIATION ON THE DYNAMICAL PROPERTIES OF WEAKLY BOUND ELECTRONS}

\author{
P. AVAN, C. COHEN-TANNOUDJI, J. DUPONT-ROC and C. FABRE \\ Laboratoire de Spectroscopie Hertzienne de l'E.N.S. $\left({ }^{*}\right)$ et Collège de France, \\ 24, rue Lhomond, 75231 Paris Cedex 05, France
}

(Reçu le 18 mars 1976, accepté le 23 avril 1976)

\begin{abstract}
Résumé. - On étudie comment le comportement d'un électron dans des champs statiques électrique ou magnétique est modifié lorsque cet électron interagit également avec une onde électromagnétique haute fréquence. Les nouvelles propriétés dynamiques de l'électron sont décrites par un hamiltonien effectif dont l'expression est établie en utilisant une description quantique du champ électromagnétique et en tenant compte des corrections relativistes jusqu'à l'ordre $1 / c^{2}$. Outre une correction de masse bien connue, on trouve que les nouvelles propriétés dynamiques de l'électron peuvent être comprises en termes de facteurs de forme électrique et magnétique qui ont une interprétation physique simple. Ces résultats généraux sont enfin appliqués à 2 cas simples : perturbation des niveaux de Rydberg d'un atome et modification des fréquences cyclotron et de précession de spin d'un électron dans un champ magnétique uniforme.
\end{abstract}

\begin{abstract}
We study how the behaviour of an electron in d.c. electric or magnetic fields changes when this electron is simultaneously interacting with a high frequency electromagnetic wave. The new dynamical properties of the electron are described by an effective hamiltonian that we derive using a quantum description of the electromagnetic field and including relativistic corrections up to order $1 / c^{2}$. Besides a well-known mass-shift correction, one finds that the new dynamical properties of the electron can be described in terms of electric and magnetic form factors which have a simple physical interpretation. Finally, these general results are applied to 2 simple cases : perturbation of atomic Rydberg states and modification of the cyclotron and spin precession frequencies of an electron in a static homogeneous magnetic field.
\end{abstract}

1. Introduction. - 1.1 Motivations FOR THIS WORK. - The initial motivation of this work was to extend some previous calculations dealing with the effect of a non-resonant irradiation on a neutral atomic system.

It is well known for example that atomic energy levels are shifted when atoms are interacting with a non-resonant light beam. These so called light-shifts have been mainly studied in cases where the main part of the effect is due to virtual transitions to a quasiresonant state $[1,2,3,4,5]$ or to a few excited states $[6,7,8]$. But such an approximation is not always possible. For example, when atoms in highly excited states (Rydberg states) interact with an intense light beam having a frequency much higher than the

$\left(^{*}\right)$ Associé au C.N.R.S. spacing and the ionization energy of these states, it is necessary to consider the effect of virtual transitions to all atomic states including the continuum. One can ask if it would not be possible in this case to make a convenient approximate evaluation of the infinite sum appearing in the second order term of the perturbation series, which would not require the knowledge of all atomic oscillator strengths.

Another example of a problem which motivated this work is the modification of the $g$ factor of an atomic state under the influence of non-resonant RF irradiation (having a frequency $\omega$ much higher than the spin precession frequency $\left.\omega_{0}\right)[9,10,11]$. The important point is that one always finds a reduction of the $g$ factor, never an enhancement. But it is well known that the $g$ factor of a free electron is increased above the value 2 predicted by the Dirac equation when one takes into account the coupling with the 
quantized electromagnetic field vacuum : the $g-2$ anomaly [12] is positive. What is the origin of this difference of sign ? Is it due to the fact that one considers in the first case a neutral atomic system, in the second one a charged particle ? Or to the fact that the system is coupled in the first case to a filled mode of the electromagnetic field, in the second one, to the vacuum ? A first step to answer these questions is to determine precisely how the cyclotron and the spin precession frequencies are modified by high frequency irradiation.

We present in this paper a simple approach to these various problems. We consider an electron weakly bound in a d.c. electric field (for example, an electron in a Rydberg state), or orbiting in a d.c. magnetic field, and we try to understand the perturbation of the energy levels of such an electron, when irradiated by a high frequency electromagnetic wave, in terms of corrections to its dynamical properties (corrections to its mass, appearance of electric and magnetic form factors, ...). In other words, we try to understand how the high frequency vibration of the electron (in the incident wave) changes the slow motion of this electron (in the applied d.c. fields).

1.2 CONNECTIONS WITH PREVIOUS WORKS. - The possibility of irradiating electrons with intense laser beams has already stimulated a lot of theoretical works. Most of these treatments consider essentially free electrons. Their purpose is to investigate some nonlinear effects appearing in the scattering by the electron of the high intensity incident radiation : harmonic production, intensity dependent frequency shift in Compton scattering, ... or to study some associated effects on the electron itself : mass-shift of an electron in a plane wave, deviation by a high intensity standing wave (Kapitza-Dirac effect), reflection and refraction of free electrons in spatially inhomogeneous laser beams, ... (An important list of references may be found in reference [13] which is a review paper on the subject.)

We will not consider here such scattering problems. Our interest lies in the modifications induced by the light irradiation on the dynamical properties of an electron which is supposed to be weakly bound in a d.c. external field (the electron wave function is confined to a sufficiently small region of space so that one can neglect any spatial variation of the laser intensity within this region and all the corresponding intensity gradient forces). We will show that the electron moves in the static fields as if it had an effective mass greater than $m$ by an amount which is nothing other than the mass shift derived previously for a free electron interacting with a strong electromagnetic wave $\left({ }^{1}\right)$. But we will also derive a lot of other corrections, which are not related to such a mass

( $\left.{ }^{1}\right)$ Let us emphasize however that such a mass shift correction is only valid for a weakly bound electron (see discussion of section 3.1 i). shift, and which may be interpreted in terms of electric and magnetic form factors induced by the incident wave and modifying the coupling of the electron to the static fields. We will discuss in detail these corrections, their physical interpretation and their possible observation.

Most of the previous theoretical treatments mentioned above make use of the exact solutions of the Dirac equation for an electron in a classical plane electromagnetic wave (Volkov's states, see ref. [14]), or of the Green function associated with such a wave equation [15]. Some progress has recently been made in finding exact solutions of the Dirac equation for an electron in a quantized plane wave $[16,17]$, or in a quantized plane wave and in a constant magnetic field [18]; the motivation of these calculations being essentially to calculate the radiation absorbed or emitted by such an electron.

In the present paper, we do not start from Volkov's solutions, although it would be possible to study the perturbation of such solutions by the external static fields in order to get the modified response of the electron to these static. fields (such an approach is suggested in reference [19] but we have not found in the litterature any further progress in this direction). We have preferred to work in the non-relativistic limit and use the Foldy-Wouthuysen hamiltonian for describing (up to order $v^{2} / c^{2}$ ) the coupling of the electron with the static fields and the incident wave. We then derive an effective hamiltonian giving the new perturbed Bohr frequencies associated with the slow motion of the electron in the static fields. We have chosen such an approach for three reasons : (i) It gives the modifications of the dynamical properties of the electron as correction terms to a nonrelativistic hamiltonian and the physical interpretation is straightforward. Furthermore, the corrections can be readily evaluated using non-relativistic (2-components) wave functions. (ii) From an experimental point of view, the higher order terms appear to be completely negligible. (iii) It may seem questionable to compute higher order terms from exact solutions of Dirac equation and not from the Q.E.D. formalism.

Let us finally explain why we have chosen a quantum description of the incident wave, rather than a classical one. A quantum treatment of the electromagnetic field gives simultaneously the effect of the coupling with the photons of the mode (stimulated effects) and the effect of the coupling with the empty mode (spontaneous effects). In this paper, we will focus on the stimulated effects. But we will come back later on to the spontaneous effects in connection with the $g-2$ anomaly. Such an approach will give us the possibility of comparing the 2 types of effects and to get some physical insight into the $g-2$ problem which has received a lot of attention [20].

1.3 OUTLINE OF THE PAPER. - In section 2, we present the effective hamiltonian method and we discuss the classification and the order of magnitude 
of the various terms of this hamiltonian. These terms are explicitly given in section 3 , and one shows how they can be classically interpreted. Finally, in section 4 , we apply our results concerning the effect of high frequency irradiation to 2 cases : the perturbation of Rydberg states and the modification of the cyclotron and spin precession frequencies in a static homogeneous magnetic field.

2. General method. - 2.1 Hamiltonian OF THE SYSTEM. - Consider an electron of charge $e$, rest mass $m$, which is irradiated by an intense and monochromatic plane wave of pulsation $\omega$, wave vector $\mathbf{k}$ (with $\mathbf{k}=\mathbf{k} /|\mathbf{k}|$ ), polarization $\varepsilon$ (linear or circular), and submitted to d.c. electric and magnetic fields, described by the fields $\mathbf{E}_{0}, \mathbf{B}_{0}$ or the potentials $\mathbf{A}_{0}, \varphi_{0}$.

As mentioned above, we will treat quantummechanically the interaction with the electromagnetic fields. For the sake of simplicity, we will describe the incident electromagnetic wave (i.e.w.) by a state vector with $N$ photons in the mode $(\mathbf{k}, \varepsilon)$ and 0 in all other modes, represented by the ket $|N\rangle$. We call $a$ and $a^{\dagger}$ the annihilation and creation operators operating in this mode.

In order to describe the interaction between the electron and the different fields, we can of course use the Pauli hamiltonian. But we thus neglect several interesting relativistic effects, which may be not negligible in some cases. To take into account such effects, we will use the non-relativistic limit of the Dirac equation, computed up to 2 nd order in powers of $v / c$ : all relativistic effects up to $1 / c^{2}$ will then be described.

It is possible, by the method of Foldy and Wouthuysen, to derive from the Dirac equation the relativistic corrections to the Pauli hamiltonian. In our special case, we must slightly modify this technique, because the fields we deal with are quantized, and the corresponding operators do not commute. The final result is the same if we ignore a constant term. In the Coulomb gauge this non-relativistic hamiltonian can be written as :

$$
\begin{aligned}
\mathscr{H}_{\mathrm{FW}}=\hbar \omega a^{\dagger} a+\frac{\pi^{2}}{2 m}+e \varphi_{0}-\frac{e \hbar}{2 m} \boldsymbol{\sigma} . \mathbf{B}_{\mathrm{t}}- \\
-\frac{e \hbar^{2}}{8 m^{2} c^{2}} \nabla \cdot \mathbf{E}_{\mathrm{q}}-\frac{e \hbar}{8 m^{2} c^{2}} \boldsymbol{\sigma} \cdot\left(\mathbf{E}_{\mathrm{t}} \times \pi-\pi \times \mathbf{E}_{\mathbf{t}}\right)- \\
\quad-\frac{1}{2 m c^{2}}\left(\frac{\pi^{2}}{2 m}-\frac{e \hbar}{2 m} \boldsymbol{\sigma} \cdot \mathbf{B}_{\mathrm{t}}\right)^{2} \cdot
\end{aligned}
$$

In this expression, $\mathbf{E}_{\mathbf{t}}$ and $\mathbf{B}_{\mathbf{t}}$ are the total fields (static + plane wave),

$$
\begin{aligned}
\boldsymbol{\pi} & =\mathbf{p}-e \mathbf{A}_{0}-e \mathbf{A}_{\mathbf{r}} \\
& =\boldsymbol{\pi}_{0}-e \mathbf{A}_{\mathbf{r}}
\end{aligned}
$$

is the electron linear momentum. The radiation field vector potential $\mathbf{A}_{\mathbf{r}}$ has the following expression :

$$
\mathbf{A}_{\mathbf{r}}=\sqrt{\frac{\hbar}{2 \varepsilon_{0} \omega L^{3}}}\left(a \varepsilon \mathrm{e}^{i \mathbf{k} . \mathbf{r}}+a^{\dagger} \varepsilon^{*} \mathrm{e}^{-i \mathbf{k} . \mathbf{r}}\right)
$$

( $L^{3}$ : quantization volume). We will also use the r.m.s. value $E$ of the radiation electric field $\mathbf{E}_{\mathrm{r}}$, which is related to the number $N$ of photons by the equation :

$$
N \hbar \omega=\frac{1}{2} \varepsilon_{0} E^{2} L^{3} .
$$

This equation expresses the equality between the two expressions (quantum-mechanical and classical) of the i.e.w. energy within the volume $L^{3}$.

In the expression (2.1), the first line gives the free radiation hamiltonian and the electronic Pauli hamiltonian. In the second line the following terms appear successively : the Darwin term, the spin-orbit term, the relativistic mass correction.

Let us now separate the contributions of the different fields. $\mathscr{H}_{\mathrm{FW}}$ is then a sum of three terms :

- The free field hamiltonian $\mathfrak{H}_{\mathrm{f}}$ :

$$
\mathscr{H}_{\mathrm{f}}=\hbar \omega a^{\dagger}, a \text {. }
$$

- The electronic hamiltonian $\mathfrak{H}_{\mathrm{e}}$, describing the electron in presence of the static fields only :

$$
\begin{aligned}
\mathscr{H}_{\mathrm{e}}=\frac{\pi_{0}^{2}}{2 m}+ & e \varphi_{0}-\frac{e \hbar}{2 m} \boldsymbol{\sigma} \cdot \mathbf{B}_{0}+\frac{e \hbar^{2}}{8 m^{2} c^{2}} \Delta \varphi_{0}+ \\
& +\frac{e \hbar}{4 m^{2} c^{2}} \boldsymbol{\sigma} \cdot\left(\nabla \varphi_{0} \times \pi_{0}\right)- \\
& -\frac{1}{2 m c^{2}}\left(\frac{\pi_{0}^{2}}{2 m}-\frac{e \hbar}{2 m} \boldsymbol{\sigma} \cdot \mathbf{B}_{0}\right)^{2}
\end{aligned}
$$

- The interaction hamiltonian $\mathfrak{H}_{\mathrm{I}}$, describing the interaction between the electron and the i.e.w. splits into two terms : $\mathfrak{H}_{\mathrm{I}_{1}}$, linear in $a$ and $a^{\dagger}, \mathfrak{H}_{\mathrm{I}_{2}}$ containing all higher order terms in $a$ and $a^{\dagger}$, which are respectively given, when one takes into account only terms up to $\frac{1}{c^{2}}\left(\right.$ with $\left.\left|\mathbf{B}_{\mathrm{r}}\right|=\frac{\omega}{c}\left|\mathbf{A}_{\mathrm{r}}\right|\right)$, by the following expressions :

$$
\begin{gathered}
\mathscr{H}_{\mathrm{I}_{\mathrm{r}}}=-\frac{e}{m} \mathbf{A}_{\mathrm{r}} \cdot \pi_{0}-\frac{e \hbar}{2 m} \sigma \cdot \mathbf{B}_{\mathrm{r}}+\frac{e^{2} \hbar}{4 m^{2} c^{2}} \boldsymbol{\sigma} .\left(\mathbf{E}_{0} \times \mathbf{A}_{\mathrm{r}}\right)- \\
-\frac{e \hbar}{4 m^{2} c^{2}} \boldsymbol{\sigma} \cdot\left(\mathbf{E}_{\mathrm{r}} \times \pi_{0}\right)+\frac{e}{2 m^{2} c^{2}} \times \\
\times\left\{\mathbf{A}_{\mathrm{r}} \cdot \pi_{0}\left(\frac{\pi_{0}^{2}}{2 m}-\frac{e \hbar}{2 m} \sigma . \mathbf{B}_{0}\right)+\text { herm. conj. }\right\} \\
\mathscr{H}_{\mathrm{F}_{2}}=\frac{1}{2 m} e^{2} \mathbf{A}_{\mathrm{r}}^{2}+\frac{e^{2} \hbar}{4 m^{2} c^{2}} \boldsymbol{\sigma} \cdot\left(\mathbf{E}_{\mathrm{r}} \times \mathbf{A}_{\mathrm{r}}\right)- \\
-\frac{e^{2} \mathbf{A}_{\mathrm{r}}^{2}}{2 m^{2} c^{2}}\left(\frac{\pi_{0}^{2}}{2 m}-\frac{e \hbar}{2 m} \boldsymbol{\sigma} \cdot \mathbf{B}_{0}\right)- \\
-\frac{e^{2}}{8 m^{3} c^{2}}\left(2 \mathbf{A}_{\mathrm{r}} \cdot \pi_{0}-e \mathbf{A}_{\mathrm{r}}^{2}\right)^{2} .
\end{gathered}
$$


2.2 EfFective hamiltonian method. - We first consider the energies of the system electron + photons without any mutual interaction. Let $\varepsilon_{\alpha}, \varepsilon_{\beta}, \ldots$, be the eigenvalues of the electronic hamiltonian $\mathscr{H}_{\mathrm{e}}$, corresponding to eigenstates $|\alpha\rangle,|\beta\rangle \ldots$ The hypothesis of a high frequency, non-resonant field can be specified in the following way :

For every pair $\alpha, \beta$ :

$$
\left|\varepsilon_{\alpha}-\varepsilon_{\beta}\right| \ll \hbar \omega
$$

The energy diagram is represented on figure 1 . As we will see later, the relevant states bunch into well separated multiplicities $\varepsilon_{N}$, corresponding to tensorial product states $|N\rangle \otimes|\alpha\rangle$.

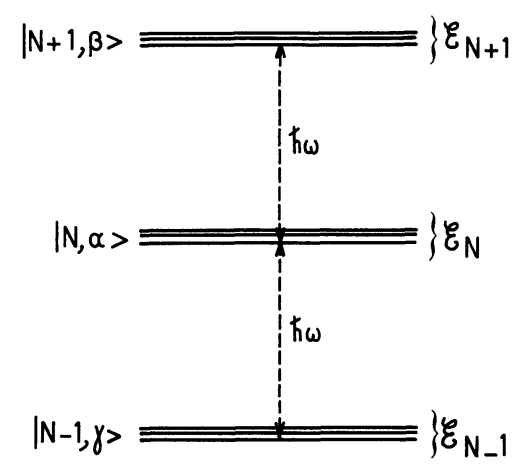

FIG. 1. - Energy diagram of the system electron + photons without interaction ( $N:$ number of photons, $\alpha \beta \gamma$ : electron states). The high frequency condition ensures that the multiplicities... $\varepsilon_{N-1}, \varepsilon_{N}, \varepsilon_{N+1}, \ldots$ are well separated.

The hamiltonian $\mathscr{H}_{\mathrm{I}}$, describing the interaction between the electron and the photon field, can be divided into two parts :

- an off-diagonal part, coupling multiplicities $\varepsilon_{N}$ and $\delta_{N}$, corresponding to different photon numbers ;

- a diagonal part, only operating inside the multiplicity $\varepsilon_{N}$.

We now suppose that the off-diagonal part of the coupling is small compared to the splitting between two multiplicities, i.e. :

$$
\left|\left\langle N, \alpha\left|\mathscr{H}_{\mathrm{I}}\right| N^{\prime}, \beta\right\rangle\right| \ll \hbar \omega \text { if } N \neq N^{\prime} .
$$

It is therefore possible to apply perturbation theory to obtain the eigenfrequencies and the eigenstates of the total hamiltonian. Due to the coupling $\mathfrak{H}_{\mathrm{I}}$, these eigenstates do not correspond to a well-defined value of $N$; in the evolution of the electronic variables, some frequencies close to $\omega$ and its multiples appear, corresponding to the classical picture of an electron vibrating in the electric field of the i.e.w. Moreover, the energy splittings inside a given multiplicity, and consequently the slow motion of the electron, are modified. This modification of the dynamical electronic properties in the static fields is precisely what we call dressing of the electron, and can be interpreted in two different ways :
- In quantum language, the virtual absorption and reemission of incident photons (and the reverse process) influence the electronic behaviour in an applied static field.

- In classical language, the slow motion of the electron due to the applied fields is affected by the high frequency vibration induced by the i.e.w.

As we are mainly interested in this modification of the electron's dynamical properties, we only need the low Bohr frequencies of the system. We will now show that these frequencies can be obtained by a purely operator method, called the effective hamiltonian method. The idea is to apply a unitary transformation $U$ to the total hamiltonian in such a way that it eliminates the off-diagonal part of the coupling to a given order of perturbation. The transformed hamiltonian $\mathscr{H}_{\text {eff }}$, called effective hamiltonian, has thus the same eigenvalues as the total hamiltonian, but it only acts within a given multiplicity, i.e. operates only on electronic variables.

Before giving the explicit form of the effective hamiltonian, let us make two important remarks :

(i) The energy spectrum of any electron has not, in fact, an upperbound : rigorously speaking, it is not correct to consider that the multiplicities $\varepsilon_{N}$ are well separated since some states of $\varepsilon_{N}$ are degenerate with some states of $\varepsilon_{N^{\prime}}\left(N^{\prime} \neq N\right)$; this simply expresses the fact that real transitions are possible between electronic states under the influence of the i.e.w. We can ignore the coupling between these degenerate states provided that the corresponding matrix elements are sufficiently small. More precisely, we will suppose that the time constants associated with the resonant (or quasi-resonant) couplings are much longer than the characteristic evolution times in the applied static fields (of the order of $\left.\frac{2 \pi}{\varepsilon_{\alpha}-\varepsilon_{\beta}}\right)$. This condition must be checked in each particular case.

(ii) The effective hamiltonian method yields the eigenvalues of the total hamiltonian, but not its eigenstates. If they are needed, we must perform the inverse unitary transformation $U^{\dagger}$ on the eigenstates of $\mathcal{T}_{\text {eff }}$.

We show in appendix A (see also [21]) that the matrix elements of $\mathfrak{H}_{\text {eff }}$, up to 2 nd order of perturbation, can be expressed as :

$$
\begin{aligned}
& \left\langle N, \alpha\left|\mathscr{H}_{\text {eff }}\right| N, \beta\right\rangle=\left\langle\alpha\left|\mathcal{H}_{\mathrm{e}}\right| \beta\right\rangle+ \\
& +N \hbar \omega+\left\langle N, \alpha\left|\mathcal{H}_{\mathrm{I}}\right| N, \beta\right\rangle+ \\
& \quad+\frac{1}{2} \sum_{\substack{\gamma N^{\prime} \\
\left(N^{\prime} \neq N\right)}}\left(\frac{1}{\mathcal{E}_{N, \alpha}-\mathcal{E}_{N^{\prime}, \gamma}}+\frac{1}{\mathcal{E}_{N, \beta}-\varepsilon_{N^{\prime}, \gamma}}\right) \\
& \quad \times\left\langle N, \alpha\left|\mathscr{H}_{\mathrm{I}}\right| N^{\prime}, \gamma\right\rangle\left\langle N^{\prime}, \gamma\left|\mathcal{H}_{\mathrm{I}}\right| N, \beta\right\rangle .
\end{aligned}
$$

This expression reduces to the usual perturbation development for the diagonal elements 
$\left\langle N, \alpha\left|\mathscr{H}_{\text {eff }}\right| N, \alpha\right\rangle$. It is different for the off-diagonal elements inside $\varepsilon_{N}$ : instead of taking the usual energy fraction $\frac{1}{\varepsilon_{N, \alpha}-\varepsilon_{N^{\prime}, \gamma}}$, we take the mean value of the 2 possible fractions.

The energy denominators are more precisely written as

$$
\varepsilon_{\alpha}-\varepsilon_{\gamma}+\left(N-N^{\prime}\right) \hbar \omega
$$

We can thus take into account the inequality $(2.10)$ and expand the energy fractions in powers of $\frac{\mathcal{E}_{\alpha}-\varepsilon_{\gamma}}{\hbar \omega}$, so that the electronic energies do not appear any more in the denominator.

So far, $\mathscr{H}_{\text {eff }}$ is only defined by its matrix elements.
We now show that we can obtain a pure operator form of $\mathscr{H}_{\mathrm{eff}}$, only acting on electronic variables.

2.3 OPERATOR FORM OF THE ELECTRONIC EFFECTIVE HAMILTONIAN. - Let us disentangle in $\mathscr{H}_{\mathbf{I}_{1}}$ and in $\mathscr{H}_{\mathbf{I}_{2}}$ the electronic and field operators :

$$
\begin{aligned}
\mathscr{H}_{\mathrm{I}_{1}}= & V^{+} a^{\dagger}+V^{-} a \\
\mathscr{H}_{\mathrm{I}_{2}}= & V^{++}\left(a^{\dagger}\right)^{2}+V^{+-} a^{\dagger} a+ \\
& \quad+V^{-+} a a^{\dagger}+V^{--}(a)^{2}+V_{3}
\end{aligned}
$$

$V_{3}$ contains the terms of (2.9) which are proportional to $e^{3}$ and $e^{4}$, whereas the four first terms of $\mathscr{H}_{1}$ are proportional to $e^{2}$. The electronic operators have the following expressions :

$$
\begin{aligned}
& V^{-}=\sqrt{\frac{\hbar}{2 \varepsilon_{0} \omega L^{3}}}\left[-\frac{e}{m} \mathrm{e}^{i \mathbf{k} . \mathbf{r}} \boldsymbol{\varepsilon} . \pi_{0}-\frac{i e \hbar}{2 m} \mathrm{e}^{i \mathbf{k} . \mathbf{r}} \boldsymbol{\sigma} .(\mathbf{k} \times \boldsymbol{\varepsilon})+\frac{e^{2} \hbar}{4 m^{2} c^{2}} \mathrm{e}^{i \mathbf{k} . \mathbf{r}} \boldsymbol{\sigma} \cdot\left(\mathbf{E}_{0} \times \boldsymbol{\varepsilon}\right)-\right. \\
& \left.-\frac{i e \hbar \omega}{4 m^{2} c^{2}} \mathrm{e}^{i \mathbf{k} . \mathbf{r}} \boldsymbol{\sigma} .\left(\boldsymbol{\varepsilon} \times \pi_{0}\right)+\frac{e}{4 m^{2} c^{2}}\left\{\left(2 \mathrm{e}^{i \mathbf{k} . \mathbf{r}} \boldsymbol{\varepsilon} . \pi_{0}+i \hbar \mathrm{e}^{i \mathbf{k} . \mathbf{r}} \mathbf{k} \times \boldsymbol{\varepsilon}\right)\left(\frac{\pi_{0}^{2}}{2 m}-\frac{e \hbar}{2 m} \boldsymbol{\sigma} . \mathbf{B}_{0}\right)+\text { herm. conj. }\right\}\right] \\
& V^{+}=\left(V^{-}\right)^{\dagger} \\
& V^{-+}=\frac{\hbar}{2 \varepsilon_{0} \omega L^{3}}\left[\frac{e^{2}}{2 m}+\frac{i e^{2} \hbar \omega}{4 m^{2} c^{2}} \boldsymbol{\sigma} .\left(\boldsymbol{\varepsilon} \times \varepsilon^{*}\right)-\frac{e^{2}}{2 m^{2} c^{2}}\left(\frac{\pi_{0}^{2}}{2 m}-\frac{e \hbar}{2 m} \boldsymbol{\sigma} . \mathbf{B}_{0}\right)-\right. \\
& -\frac{e^{2}}{8 m^{3} c^{2}}\left(2 \varepsilon . \pi_{0}+i \hbar \sigma .(\mathbf{k} \times \varepsilon)\right)\left(2 \varepsilon^{*} . \pi_{0}-i \hbar \sigma .\left(k \times \varepsilon^{*}\right)\right)
\end{aligned}
$$

$V^{+-}$is obtained from $V^{-+}$be changing $\varepsilon$ to $\varepsilon^{*}$ and $i$ to $-i$.

The $V^{--} a^{2}$ and $V^{++}\left(a^{\dagger}\right)^{2}$ terms, which couple the $\varepsilon_{N}$ and $\varepsilon_{N \pm 2}$ multiplicities, give rise to 4-photon processes ( 2 absorptions, 2 emissions). These terms have the same order of magnitude as 4th order perturbation terms, and we can therefore neglect them. For the same reason, taking into account the $V_{3}$ term in $\mathcal{H}_{\mathbf{I}_{2}}$ is inconsistent with a second order perturbation treatment, and we will therefore neglect $V_{3}$.

Now, if we develop the energy fractions up to 2 nd order in powers of $\left(\frac{\varepsilon_{\alpha}-\varepsilon_{\gamma}}{\hbar \omega}\right)$, we obtain the following expression of the effective hamiltonian, where all field operators have disappeared :

$$
\begin{aligned}
& \mathscr{H}_{\mathrm{eff}}=\mathscr{H}_{\mathrm{e}}+N V^{+-}+(N+1) V^{-+}-\frac{N}{\hbar \omega}\left[V^{-}, V^{+}\right]-\frac{1}{\hbar \omega} V^{-} V^{+}- \\
& -\frac{N}{2 \hbar^{2} \omega^{2}}\left(\left[V^{-},\left[V^{+}, \mathscr{H}_{\mathrm{e}}\right]\right]+\left[V^{+},\left[V^{-}, \mathscr{H}_{\mathrm{e}}\right]\right]\right)-\frac{1}{2 \hbar^{2} \omega^{2}}\left(\left[\mathcal{H}_{\mathrm{e}}, V^{-}\right] V^{+}-V^{-}\left[\mathcal{H}_{\mathrm{e}}, V^{+}\right]\right)
\end{aligned}
$$

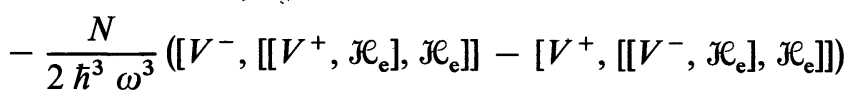

$$
\begin{aligned}
& -\frac{1}{2 \hbar^{3} \omega^{3}}\left(\mathscr{H}_{\mathrm{e}}\left[\mathscr{H}_{\mathrm{e}}, V^{-}\right] V^{+}-V^{-}\left[\mathscr{H}_{\mathrm{e}}, V^{+}\right] \mathscr{H}_{\mathrm{e}}+V^{-} \mathscr{H}_{\mathrm{e}}\left[\mathscr{H}_{\mathrm{e}}, V^{+}\right]-\left[\mathscr{H}_{\mathrm{e}}, V^{-}\right] \mathcal{H}_{\mathrm{e}} V^{+}\right) \text {. }
\end{aligned}
$$

The effective hamiltonian (2.16) exhibits two kinds of terms :

(i) $N$-independent terms, which describe the contribution of the considered $(\mathbf{k}, \varepsilon)$ mode to spontaneous effects, due to the coupling of the electron with the vacuum. We will focus on the physical interpretation of these terms in a subsequent paper.

(ii) Terms proportional to $N$, which are proportional to the light intensity and describe effects stimulated by the i.e.w. As mentioned in the introduction, in the present paper we will only consider these terms (we ignore all spontaneous effects) $\left({ }^{2}\right)$.

$\left({ }^{2}\right)$ Strictly speaking, all modes contribute to spontaneous effects and should be considered. The corresponding effects can be accounted for by introducing the usual radiative corrections in the electronic hamiltonian (see for example ref. [22]). Such a procedure renormalizes the unperturbed hamiltonian and also slightly modifies the stimulated terms of the effective hamiltonian. To simplify, we will discard these radiative corrections to the stimulated terms. 
The expression (2.16) of $\mathscr{H}_{\text {eff }}$ contains terms like : $V^{+} \mathcal{H}_{\mathrm{e}} V^{-} /(\hbar \omega)^{2}$ : such terms take into account not only the effect of a virtual transition via $\varepsilon_{N \pm 1}$, but also the effect of an intermediate evolution inside $\varepsilon_{N \pm 1}$ due to $\mathfrak{H}_{\mathrm{e}}$, i.e. due to the applied static fields : these terms describe the dressed electron polarization under the effect of the applied static fields.

The expressions (2.14) and (2.15) of $V^{+}$and $V^{-}$ contain a lot of propagation factors $\mathrm{e}^{ \pm i k r}$. These factors are important and we cannot make the dipole approximation $\mathrm{e}^{i \mathbf{k} . \mathbf{r}}=1$, because the development of these factors gives powers of $i k r=i \omega r / c$, proportional to $1 / c, 1 / c^{2}, \ldots$ : we must keep such terms since we want to make a consistent calculation up to 2 nd order in powers of $1 / c$. As a matter of fact, this factor can be exactly accounted for, because it is nothing else than a translation operator in momentum space.

To summarize, we can say that the final expression of $\mathcal{H}_{\text {eff }}$ is obtained by making several developments :

(i) Development in powers of $1 / c$ up to $1 / c^{2}$ in the Foldy-Wouthuysen hamiltonian and in the calculation of the effective hamiltonian.

(ii) Perturbation development up to 2nd order in $E$ (r.m.s. electric field of the i.e.w.) in the expression (2.12) of $\mathfrak{X}_{\text {eff }}$.

(iii) Development of the energy fraction $\frac{1}{\varepsilon_{\alpha}-\varepsilon_{\gamma} \pm \hbar \omega}$ in powers of $\mathfrak{H}_{\mathrm{e}} / \hbar \omega$.

A great number of terms therefore appear in $\mathfrak{H}_{\text {eff }}$ : we then need a precise determination of their order of magnitude (in terms of the characteristic parameters of the problem) in order to keep in the hamiltonian all the terms up to a well defined order of magnitude, and only these ones.

2.4 Classification OF THE DIFFERENT TERMS. The problem we deal with depends on a small number of independent characteristic energies :

- The coupling between the electron and the plane wave can be specified by the vibrational kinetic energy $\varepsilon_{\mathrm{v}}$ of the electron in the classical electric field of the i.e.w. (see eq. (B.7) of appendix B) :

$$
\varepsilon_{\mathrm{v}}=\frac{e^{2} E^{2}}{2 m \omega^{2}} .
$$

- The coupling between the electron and the applied static fields is characterized by the energy $\varepsilon_{\mathrm{b}}$; the expression of $\varepsilon_{b}$ depends on the particular problem we are interested in: it can be for example $\hbar \omega_{c}$ $\left(\omega_{\mathrm{c}}\right.$ : cyclotron frequency) in the case of a static magnetic field, or $\alpha^{2} m c^{2} / 2 n^{2}$ in the case of a Coulomb field, etc...

- The free photon field and the free electron are characterized by the energies $\hbar \omega$ and $m c^{2}$.

It is easy to see that the order of magnitude of each term in $V^{-}$or $V^{+}$, and hence in the effective hamiltonian can be expressed as a function of these four characteristic energies. For example, the second term in $N V^{-+}$(eq. (2.15)) has the following order of magnitude :

$$
\begin{aligned}
\frac{i e^{2} \hbar^{2} N}{8 \varepsilon_{0} L^{3} m^{2} c^{2}} \sigma .\left(\varepsilon \times \varepsilon^{*}\right) & \approx \frac{e^{2} \hbar^{2} N}{\varepsilon_{0} m^{2} c^{2} L^{3}}= \\
& =\frac{e^{2} \hbar E^{2}}{m^{2} c^{2} \omega} \approx \varepsilon_{\mathrm{v}} \frac{\hbar \omega}{m c^{2}}
\end{aligned}
$$

In order to be sure of the convergence of the different developments which appear in the calculation of the effective hamiltonian, we will suppose that the following conditions are fulfilled :

(i) High frequency condition :

- well separated multiplicities i.e. :

$$
\varepsilon_{\mathrm{b}} \ll \hbar \omega,
$$

- negligible real transitions.

(ii) Weak coupling condition :

$$
\mathcal{E}_{\mathrm{v}} \ll \hbar \omega \text {. }
$$

(iii) Non-relativistic condition for the photon energy :

$$
\hbar \omega \ll m c^{2}
$$

which implies, according to (2.19) and (2.20), that all the energies involved in the problem are also small compared to $m c^{2}$.

With the help of these inequalities, we are now able to classify the different terms in $\mathscr{H}_{\text {eff }}$. We need now some criteria to stop the different developments in the final expression of $\mathcal{H}_{\text {eff }}$. In this paper :

- we compute all terms in $1 / c$ up to $1 / c^{2}$,

- we keep linear terms with respect to $\varepsilon_{v}$,

- we keep linear terms with respect to $\varepsilon_{\mathrm{b}}$ (linear response to the applied fields), but also the major quadratic terms, of the order of $\varepsilon_{b}^{2} / m c^{2}, \varepsilon_{v} \varepsilon_{b}^{2} /(\hbar \omega)^{2}$. We will neglect the smallest ones, of the order of $\varepsilon_{\mathrm{v}} \varepsilon_{\mathrm{b}}^{2} / \hbar \omega m c^{2}$.

3. Explicit form of the effective hamiltonian-physical interpretation of the results. - In this section, we give the explicit form of the effective hamiltonian, more precisely of the stimulated terms of this hamiltonian. As the general method for calculating and classifying these terms has been explained in detail in the previous section, we don't give here the details of the calculation. We prefer to focus on physical discussions and to show how each term of this effective hamiltonian can be classically interpreted as a result of the vibration of the electron and of its spin in the i.e.w. Some results concerning the classical motion of an electron in a plane wave, and which are referred to in the following discussion, are recalled in appendix $B$.

We will consider separately the spin independent and the spin dependent terms of $\mathfrak{J}_{\text {eff }}$. 
3.1 SPIN INDEPENDENT PART OF $\mathfrak{H}_{\text {eff }}: \mathfrak{H}_{\text {eff }}^{\mathrm{I}}-\mathrm{We}$ find for $\mathcal{H}_{\text {eff }}^{\mathrm{I}}$ the following expression :

$$
\begin{aligned}
\mathscr{H}_{\mathrm{eff}}^{\mathrm{I}}=m c^{2} & +\frac{\pi_{0}^{2}}{2 m}+e \varphi_{0}+\frac{e \hbar^{2} \Delta \varphi_{0}}{8 m^{2} c^{2}}-\frac{\pi_{0}^{4}}{8 m^{3} c^{2}}+ \\
& +\varepsilon_{\mathrm{v}}-\frac{\varepsilon_{\mathrm{v}}}{m c^{2}} \frac{\pi_{0}^{2}}{2 m}+ \\
& +e \varphi_{0}^{\prime}-\frac{e}{2 m}\left(\mathbf{A}_{0}^{\prime} \cdot \pi_{0}+\pi_{0} \cdot \mathbf{A}_{0}^{\prime}\right)+ \\
& +W_{\mathrm{p}}+W_{\mathrm{d}}+W_{\mathrm{p}}^{\prime} .
\end{aligned}
$$

Where $\varphi_{0}^{\prime}, \mathbf{A}_{0}^{\prime}, W_{\mathrm{p}}, W_{\mathrm{d}}, W_{\mathrm{p}}^{\prime}$ are defined and discussed below (see eq. (3.3), (3.4), (3.6), (3.7), (3.8), (3.11)).

The first line of (3.1) does not depend on the i.e.w. It represents the well known spin independent hamiltonian of an electron interacting with the static potentials $\mathbf{A}_{0}, \varphi_{0}$ (we have discarded a small term proportional to $\mathbf{B}_{0}^{2}$ ). The three last lines of (3.1) represent corrections due to the vibration of the electron in the i.e.w. and can be associated with three different physical processes.

(i) The vibrating electron has an effective mass greater than $m$. - The two terms of the second line of (3.1) represent corrections (to $m c^{2}$ and $\pi_{0}^{2} / 2 m$ ) which are easily interpreted as resulting from a change of the electron mass :

$$
m \rightarrow m_{\mathrm{eff}}=m+\frac{\varepsilon_{\mathrm{v}}}{c^{2}}
$$

where $\varepsilon_{\mathrm{v}}$ is the vibrational kinetic energy given in (2.17).

The mass shift $\varepsilon_{\mathrm{v}} / c^{2}=e^{2} E^{2} / 2 m \omega^{2} c^{2}$ can be considered as due to the high frequency vibration of the electron which is induced by the i.e.w. and which is superimposed on its slow motion in the static fields. This physical picture clearly shows that such a mass shift does not exist for low frequency irradiation $\left(\hbar \omega \ll \mathcal{E}_{\mathrm{b}}\right)$ since, in that case, the i.e.w. appears to the electron as a quasi-static electric field which polarizes the electronic orbit and produces a Stark-shift independent of $\omega$.

(ii) The vibrating electron averages the applied static fields in a small region around its mean position. - The quantities $\varphi_{0}^{\prime}$ and $\mathbf{A}_{0}^{\prime}$ appearing in the third line of (3.1) satisfy :

$$
\begin{aligned}
\varphi_{0}^{\prime} & =\frac{e^{2} E^{2}}{2 m^{2} \omega^{4}}(\varepsilon \cdot \nabla)\left(\varepsilon^{*} . \nabla\right) \varphi_{0} \\
\nabla \times \mathbf{A}_{0}^{\prime} & =\frac{e^{2} E^{2}}{2 m^{2} \omega^{4}}(\varepsilon \cdot \nabla)\left(\varepsilon^{*} \cdot \nabla\right) \mathbf{B}_{0} .
\end{aligned}
$$

The interpretation of the corresponding terms of (3.1) is quite simple. In the electric field of the i.e.w., the electron vibrates around its mean position $\mathbf{r}_{0}$ with a frequency $\omega$ and an amplitude of the order of $e E / m \omega^{2}$. Consequently, it averages the applied static fields in a small region around $\mathbf{r}_{0}$ having linear dimensions of the order of $e E / m \omega^{2}$.
More precisely, consider for example the electrostatic energy of the electron which can be written as $e \varphi_{0}\left(\mathrm{r}_{0}+\rho\right)$ where $\rho$ is the deviation from the average position $\mathbf{r}_{0}$. A Taylor expansion of $e \varphi_{0}\left(\mathbf{r}_{0}+\boldsymbol{\rho}\right)$ gives :

$$
\begin{aligned}
e \varphi_{0}\left(\mathbf{r}_{0}+\boldsymbol{\rho}\right)= & e \varphi_{0}\left(\mathbf{r}_{0}\right)+e(\boldsymbol{\rho} . \nabla) \varphi_{0}\left(\mathbf{r}_{0}\right)+ \\
& +\frac{1}{2} e(\boldsymbol{\rho} . \nabla)(\boldsymbol{\rho} . \nabla) \varphi_{0}\left(\mathbf{r}_{0}\right)+\cdots
\end{aligned}
$$

Using the expression of $\rho$ calculated in appendix B (see eq. (B.6)), and averaging over one period $2 \pi / \omega$ of the i.e.w., one finds that the first order correction vanishes $(\overline{\boldsymbol{\rho}}=0)$ and that the second order one reduces to $e \varphi_{0}^{\prime}$ where $\varphi_{0}^{\prime}$ is given by (3.3).

A similar calculation shows that the electron sees an average magnetic field which is

$$
\mathbf{B}_{0}+\frac{e^{2} E^{2}}{2 m^{2} \omega^{4}}(\varepsilon \cdot \nabla)\left(\varepsilon^{*} \cdot \nabla\right) \mathbf{B}_{0} .
$$

Such a correction in $\mathbf{B}_{0}$ is obtained by replacing $\mathbf{A}_{0}$ by $\mathbf{A}_{0}+\mathbf{A}_{0}^{\prime}$, where $\boldsymbol{\nabla} \times \mathbf{A}_{0}^{\prime}$ satisfies (3.4), so that the kinetic energy term becomes

$$
\left(\mathbf{p}-e \mathbf{A}_{0}-e \mathbf{A}_{0}^{\prime}\right)^{2} / 2 m=\left(\pi_{0}-e \mathbf{A}_{0}^{\prime}\right)^{2} / 2 m .
$$

The lowest order correction in $\mathbf{A}_{0}^{\prime}$ is

$$
-e\left(\boldsymbol{\pi}_{0} \mathbf{A}_{0}^{\prime}+\mathbf{A}_{0}^{\prime} \pi_{0}\right) / 2 m,
$$

i.e. the last term of the fourth line of (3.1) (the $\mathbf{A}_{0}^{\prime 2}$ term does not appear in $\mathcal{H}_{\text {eff }}^{\mathrm{I}}$ since it is fourth order in $E$ ).

(iii) The vibration of the electronic charge gives rise to a small orbital magnetic moment which is coupled to the static fields. - The 2 terms $W_{\mathrm{p}}$ and $W_{\mathrm{d}}$ of the last line of (3.1) are given by :

where :

$$
W_{\mathrm{p}}=-\boldsymbol{\mu} \cdot \mathbf{B}_{0}
$$

and

$$
\boldsymbol{\mu}=i \varepsilon \times \varepsilon^{*} \frac{e^{3} E^{2}}{2 m^{2} \omega^{3}}
$$

$$
W_{\mathrm{d}}=\frac{e^{4} E^{2}}{2 m^{3} \omega^{4}}\left[\mathbf{B}_{0}^{2}-\left(\boldsymbol{\varepsilon} \cdot \mathbf{B}_{0}\right)\left(\varepsilon^{*} \cdot \mathbf{B}_{0}\right)\right]
$$

$W_{\mathrm{p}}$ is proportional to $B_{0}$ (paramagnetic term), $W_{\mathrm{d}}$ to $B_{0}^{2}$ (diamagnetic term). They can be interpreted in the following way :

The vibrating charge has an orbital magnetic moment :

$$
\mathcal{M}_{\mathrm{v}}=\frac{e}{2 m}(\rho \times m \mathrm{v})=\frac{e}{2}(\rho \times v)
$$

which can be calculated from the expressions of $\rho$ and $\mathbf{v}$ given in appendix B (eq. (B .8) and (B.9)). When averaging $\mathcal{M}_{\mathrm{v}}$ over one period $2 \pi / \omega$ of the i.e.w. one gets (neglecting terms in $B_{0}^{2}$ ) :

$$
\begin{aligned}
{\overline{\aleph_{v}}}_{\mathrm{v}}=i \varepsilon & \times \varepsilon^{*} \frac{e^{3} E^{2}}{2 m^{2} \omega^{3}}+\frac{e^{4} E^{2}}{2 m^{3} \omega^{4}} \times \\
& \times\left[\left(\boldsymbol{\varepsilon} \cdot \mathbf{B}_{0}\right) \varepsilon^{*}+\left(\varepsilon^{*} . \mathbf{B}_{0}\right) \varepsilon-2 \mathbf{B}_{0}\right]
\end{aligned}
$$


The first term of (3.10) coincides with (3.7) and represents the $B_{0}$-independent part of $\mathcal{M}_{\mathrm{v}}$. Its coupling with $\mathbf{B}_{0}$ gives just the $W_{\mathrm{p}}$ term of (3.1) : $\boldsymbol{\mu}$ is parallel to $\kappa\left(\varepsilon \times \varepsilon^{*}\right.$ is proportional to $\left.\kappa\right)$ and vanishes for linear polarization $\left(\varepsilon \times \varepsilon^{*}=0\right.$ when $\left.\varepsilon=\varepsilon^{*}\right)$. This is obvious since in that case the electron vibrates along a straight line and $\rho$ and $\mathbf{v}$ are parallel (see appendix B). For elliptical polarization of the i.e.w., the electron moves along an ellipse in a plane perpendicular to $\kappa$ and $\mu$ gets a non-zero value, proportional to the area of the ellipse. One can easily show that $\mu$ is maximum for a circular polarization of the i.e.w. in which case the electron moves along a circle.

The second term of (3.10) represents the corrections to $\mu$ associated with the modifications of the electronic motion induced by $\mathbf{B}_{0}$. This term is different from zero even for linear polarization. This is due to the fact that the magnetic force $e \mathbf{v} \times \mathbf{B}_{0}$ transforms the rectilinear motion of the electron into an elliptical one (see appendix B), giving rise to a magnetic moment proportional to $B_{0}$. The coupling of the last term of (3.10) to the static magnetic field (integrated from 0 to $B_{0}$, which gives rise to a factor $1 / 2$ ) reproduces the $W_{\mathrm{d}}$ term of (3.1).

The last term $W_{\mathrm{p}}^{\prime}$ of (3.1) may be written as :

$$
\begin{array}{r}
W_{\mathbf{p}}^{\prime}=\boldsymbol{\mu} \cdot \boldsymbol{\kappa}\left[\mathbf{B}_{0} \cdot \frac{\pi_{0}}{m c}-\left(\kappa \cdot \mathbf{B}_{0}\right)\left(\kappa \cdot \frac{\pi_{0}}{m c}\right)-\right. \\
\left.-\left(\kappa \cdot \frac{\pi_{0}}{m c}\right)\left(\kappa \cdot \mathbf{B}_{0}\right)\right]
\end{array}
$$

$W_{\mathrm{p}}^{\prime}$, which is smaller than $W_{\mathrm{p}}$ by a factor of the order of $\sqrt{\varepsilon_{\mathrm{b}} / m c^{2}} \sim v / c$, represents kinematic relativistic corrections to $W_{\mathrm{p}}$. These corrections can be computed classically when one takes into account the Doppler effect, the aberration, and the variation of the electric field intensity experienced by the electron moving with the (slow) velocity $\pi_{0} / m$.

3.2 SPIN DEPENDENT PART of $\mathscr{H}_{\text {eff }}: \mathscr{H}_{\text {eff }}^{\mathrm{II}}-\mathrm{We}$ find for $\mathcal{H}_{\text {eff }}^{\mathrm{II}}$ the following expression :

$$
\begin{aligned}
& \mathscr{H}_{\text {eff }}^{I I}=-\frac{e \hbar}{2 m} \boldsymbol{\sigma} \cdot \mathbf{B}_{0}-\frac{e \hbar}{4 m^{2} c^{2}} \boldsymbol{\sigma} \cdot\left(\mathbf{E}_{0} \times \pi_{0}\right)+\frac{e \hbar}{4 m^{2} c^{2}}\left(\frac{\pi_{0}^{2}}{2 m} \boldsymbol{\sigma} \cdot \mathbf{B}_{0}+\boldsymbol{\sigma} \cdot \mathbf{B}_{0} \frac{\pi_{0}^{2}}{2 m}\right)+ \\
& +\frac{\varepsilon_{\mathrm{v}}}{2 m c^{2}} \frac{e \hbar}{2 m}\left\{2\left(\boldsymbol{\kappa} . \mathbf{B}_{0}\right)(\boldsymbol{\kappa} . \boldsymbol{\sigma})+\left(\boldsymbol{\varepsilon}^{*} \cdot \mathbf{B}_{0}\right)(\boldsymbol{\varepsilon} . \boldsymbol{\sigma})+\left(\boldsymbol{\varepsilon} . \mathbf{B}_{0}\right)\left(\varepsilon^{*} \cdot \boldsymbol{\sigma}\right)+\right. \\
& +2 \sigma \cdot B_{0}- \\
& -2 \sigma . B_{0}+\left(\varepsilon^{*} . B_{0}\right)(\varepsilon, \sigma)+\left(\varepsilon . B_{0}\right)\left(\varepsilon^{*} . \sigma\right)+ \\
& \left.+2\left[\left(\kappa \times \varepsilon^{*}\right) \cdot \mathbf{B}_{0}\right][(\kappa \times \varepsilon) \cdot \sigma]+2\left[(\kappa \times \varepsilon) \cdot \mathbf{B}_{0}\right]\left[\left(\kappa \times \varepsilon^{*}\right) \cdot \sigma\right]\right\} \text {. }
\end{aligned}
$$

The terms of the first line of (3.12) do not depend on $\boldsymbol{E}_{\mathbf{v}}$. They represent the coupling of the spin magnetic moment $\frac{e \hbar}{2 m} \boldsymbol{\sigma}$ to $\mathbf{B}_{0}$, the spin orbit interaction and velocity dependent mass corrections. The last four lines represent corrections due to the coupling with the i.e.w. Before discussing them, let us note that they can be rewritten simply as :

$$
-\frac{e}{2 m} \sum_{i j} \delta g_{i j} \sigma_{i} B_{0 j}
$$

where $\delta g_{i j}$ appears as an anisotropic Landé factor correction. This tensor is particularly simple for linear or circular polarization. In both cases, it is given by :

$$
\delta g_{i j}=\frac{\varepsilon_{\mathrm{v}}}{m c^{2}}\left(\kappa_{i} \kappa_{j}-2 \delta_{i j}\right) .
$$

The physical interpretation of the last 4 lines of (3.12) can be given in terms of 4 different physical processes :

(i) The spin magnetic moment is reduced as a consequence of its coupling with the magnetic field $\mathbf{B}_{\mathbf{r}}$ of the i.e.w. - The correction associated with the second line of (3.12) is due to the vibration of the spin magnetic moment $\frac{e \hbar}{2 m} \sigma$ which is driven by the magnetic field $\mathbf{B}_{\mathbf{r}}$ of the i.e.w. (this correction is obtained when replacing $V$ by $-\frac{e \hbar}{2 m} \boldsymbol{\sigma} \cdot \mathbf{B}_{\mathrm{r}}$ in the third line of (2.16)).

This explains why the terms contained in the second line of (3.12) have the same structure as the ones which describe the modification of the magnetic moment of a neutral atom interacting with a non-resonant RF field (having a frequency $\omega$ higher than the atomic Larmor frequency $\omega_{0}$ ). Their physical meaning is the same. The spin magnetic moment oscillates at frequency $\omega$ around a mean direction (see Fig. 2). The length of

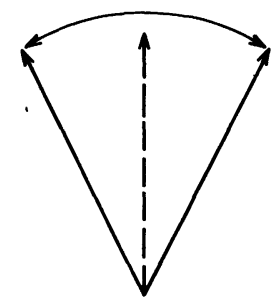

FIG. 2. - The oscillation of the spin magnetic moment in the incident wave leads to a smaller effective moment (dashed arrow).

the magnetic moment does not change during this oscillation but, as its direction is smeared out over a 
finite angle, its average value over a period $2 \pi / \omega$ is reduced and this reduces the coupling of the spin with an applied static field $\mathbf{B}_{0}$. The effect is of course anisotropic since it depends on the relative directions of $\mathbf{B}_{0}$ and $\mathbf{B}_{\mathbf{r}}$.

Remark : When the polarization is circular or elliptical, the magnetic coupling $-\frac{e \hbar}{2 m} \sigma . \mathbf{B}_{\mathbf{r}}$, gives rise to another term in $\mathcal{J}_{\text {eff }}^{\mathrm{II}}$ which can be written for right circular polarization as :

$$
-\frac{e^{2} \hbar E^{2}}{4 m^{2} c^{2} \omega} \boldsymbol{\sigma} \cdot \mathbf{\kappa} \text {. }
$$

This term is well known for neutral atoms and corresponds to the coupling of the spin with the fictitious d.c. magnetic field associated with the rotating magnetic field $\mathbf{B}_{\mathbf{r}}$ [23]. A simple classical derivation of this effect is given in appendix $\mathbf{C}$.

We have not written (3.15) in (3.12), since this term is exactly cancelled by another one which will be discussed later on (and which does not exist for neutral atoms).

The three last lines of (3.12) represent spin-dependent interactions which do not exist for a neutral particle since they are direct consequences of the vibration of the charged particle in the i.e.w.

(ii) The vibrating electron has a greater mass and, consequently, a smaller $g$ factor. - The third line of (3.12) may be interpreted as a correction resulting from the substitution of

$$
m_{\mathrm{eff}}=m+\frac{\varepsilon_{\mathrm{v}}}{c^{2}}
$$

to $m$ in the electron $g$ factor $e / m$ appearing in the first term of $\mathcal{J U}_{\text {eff }}^{\mathrm{II}}$.

(iii) The electron, vibrating in $\mathbf{E}_{\mathrm{r}}$, sees a moving magnetic field which interacts with its spin magnetic moment. - The moving magnetic field seen by the electron vibrating in $\mathbf{E}_{\mathrm{r}}$ may be written as :

$$
\mathbf{B}_{\mathrm{mot}}=-\frac{1}{c^{2}} \mathbf{v} \times \mathbf{E}_{\mathrm{r}}
$$

As $\mathbf{v}$ oscillates at the same frequency as $\mathbf{E}_{\mathbf{r}}, \mathbf{B}_{\text {mot }}$ has a d.c. component which can be calculated by replacing in (3.16) $\mathbf{v}$ and $\mathbf{E}_{\mathrm{r}}$ by the expressions (B.8) and (B.3) of appendix $B$ and by averaging over a period $2 \pi / \omega$ of the i.e.w. One gets in this way :

$$
\begin{aligned}
\overline{\mathbf{B}_{\mathrm{mot}}}=- & i \varepsilon \times \varepsilon^{*} \frac{e E^{2}}{2 m c^{2} \omega}-\frac{e^{2} E^{2}}{4 m^{2} c^{2} \omega^{2}} \times \\
& \times\left[\left(\varepsilon^{*} . \mathbf{B}_{0}\right) \varepsilon+\left(\varepsilon . \mathbf{B}_{0}\right) \varepsilon^{*}-2 \mathbf{B}_{0}\right] .
\end{aligned}
$$

The spin magnetic moment couples to $\overline{\mathbf{B}_{\text {mot }}}$ giving rise to an interaction :

$$
-\frac{1}{2} \frac{e \hbar}{2 m} \boldsymbol{\sigma} \cdot \overline{\mathbf{B}_{\mathrm{mot}}}
$$

We have added a factor $\frac{1}{2}$ because of the Thomas precession.

The first term of (3.17) gives a $\boldsymbol{B}_{0}$-independent interaction which only exists for circular or elliptical polarization and which can be written as

$$
\frac{e^{2} \hbar E^{2}}{4 m^{2} c^{2} \omega} \boldsymbol{\sigma} \cdot \boldsymbol{\kappa}
$$

in the case of right circular polarization (for which $\left.i \varepsilon \times \varepsilon^{*}=\kappa\right)$. As mentioned above, we see that this term exactly cancels (3.15). It can be seen however that such a cancellation only occurs because we have taken the electron $g$ factor exactly equal to 2 and also as a consequence of the relation $\left|\mathbf{E}_{\mathbf{r}}\right|=c\left|\mathbf{B}_{\mathrm{r}}\right|$ between $\left|\mathbf{E}_{\mathbf{r}}\right|$ and $\left|\mathbf{B}_{\mathbf{r}}\right|$ (the term (3.15) comes from the coupling with $\mathbf{B}_{\mathrm{r}}$ whereas (3.19) involves $\mathbf{E}_{\mathrm{r}}$ ). With other configurations of the electromagnetic field (such as those existing in cavities), one expects that a net effect should remain when one adds the expressions corresponding to (3.15) and (3.19).

The second term of (3.17) represents the consequences on $\overline{\mathbf{B}_{\text {mot }}}$ of the modifications of the electronic vibration induced by the static field $\mathbf{B}_{0}$. For example, for linear polarization, the first term of (3.17) vanishes $\left(E_{\mathrm{r}}\right.$ and $\mathbf{v}$ are parallel for $\left.\mathbf{B}_{0}=0\right)$, but the effect of $\mathbf{B}_{0}$ is to transform the rectilinear motion of the electron into an elliptical one (see appendix B), and $\mathbf{v} \times \mathbf{E}_{\mathrm{r}}$ gets a non-zero value. When inserted into (3.18), the last term of (3.17) gives exactly the fourth line of (3.12).

(iv) The vibrating electron can rectify the magnetic field $\mathbf{B}_{\mathbf{r}}$ of the i.e.w. - The modulation of $\mathbf{B}_{\mathbf{r}}(\mathbf{r}, t)$ due to the sinusoidal variation of the position $\mathbf{r}$ of the electron can combine with the temporal dependence of $\mathbf{B}_{\mathbf{r}}(\mathbf{r}, t)$ ( $\mathrm{e}^{ \pm i \omega t}$ factors) to give a d.c. term.

In the expansion of $\mathbf{B}_{\mathbf{r}}(\mathbf{r}, t)$ in powers of (к.r), the lowest order term depending on $\mathbf{r}$ is (see equation (B.2) of appendix B) :

$$
-\frac{E \omega}{c^{2} \sqrt{2}}(\boldsymbol{\kappa} . \mathbf{r})\left[\mathrm{e}^{-i \omega t} \mathbf{\kappa} \times \varepsilon+\mathrm{e}^{i \omega t} \mathbf{\kappa} \times \varepsilon^{*}\right] \text {. }
$$

Let us replace $\mathbf{r}$ in (3.20) by its oscillating component $\rho$ computed in appendix B (expression (B.9)). After averaging over a period $2 \pi / \omega$, one gets for the rectified magnetic field $\left(\mathbf{B}_{\mathbf{r}}\right)_{\mathrm{rect}}$ :

$$
\begin{aligned}
& \left(\mathbf{B}_{\mathrm{r}}\right)_{\mathrm{rect}}=-\frac{e^{2} E^{2}}{2 m^{2} c^{2} \omega^{2}} \times \\
& \times\left\{\left(\boldsymbol{\kappa} \times \boldsymbol{\varepsilon}^{*}\right)\left[(\mathbf{K} \times \boldsymbol{\varepsilon}) \cdot \mathbf{B}_{0}\right]+(\mathbf{\kappa} \times \boldsymbol{\varepsilon})\left[\left(\mathbf{K} \times \boldsymbol{\varepsilon}^{*}\right) . \mathbf{B}_{0}\right]\right\} .
\end{aligned}
$$


It is clear from (3.20) that such a field only exists when $\mathbf{r} . \mathbf{r} \neq 0$, i.e. when the vibration of the electron has a component along $\boldsymbol{\kappa}$. This explains why $\left(\mathbf{B}_{\mathrm{r}}\right)_{\mathrm{rect}}$ vanishes when $B_{0}=0$ since in that case the electron vibrates in a plane perpendicular to $\mathbf{K}$.

$\left(\mathbf{B}_{\mathrm{r}}\right)_{\mathrm{rec}}$ couples to the spin magnetic moment, giving rise to an interaction $-\frac{e \hbar}{2 m} \boldsymbol{\sigma} .\left(\mathbf{B}_{\mathrm{r}}\right)_{\text {rect }}$ which exactly coincides with the last line of (3.12).

To summarize the results of this section, we see that all the terms of the effective hamiltonian have a very simple physical meaning and can be quantitatively interpreted in classical terms. Let's emphasize that the spin dependent terms have a completely different structure according to whether the particle which carries the magnetic moment is charged or not. A lot of new magnetic couplings appear as a consequence of the spatial vibration of the charge and cannot be simply interpreted through a change of the electron mass. Let us explain why, at this order of the calculation $\left(\varepsilon_{v} \varepsilon_{\mathrm{b}} / m c^{2}\right)$, no spin dependent terms appear which involve the static electric field $\mathbf{E}_{\mathbf{0}}$. This is due to the fact that the electric force $e \mathbf{E}_{0}$ is static and does not affect the characteristics of the electron vibration. This is to be contrasted with the magnetic force $e \mathbf{v} \times \mathbf{B}_{0}$ which, through the sinusoidal variation of $\mathbf{v}$, can combine with $e \mathbf{E}_{\mathrm{r}}$ to change appreciably the vibration of the charge, and, consequently, the magnetic coupling of the spin.

4. Application to two simple problems. - In this section, we apply the previous general results to two particular questions :

- how atomic Rydberg states are perturbed by a high frequency non-resonant light beam ?

- how the electron cyclotron motion and its spin precession are modified by an intense electromagnetic wave ?

We have seen that $\varepsilon_{\mathrm{v}}$ is the important parameter of the problem ; let us give an order of magnitude for two experimental conditions; a focused nitrogen laser delivering a flux of $1 \mathrm{GW} / \mathrm{cm}^{2}$ or a focused $\mathrm{CO}_{2}$ laser of $1 \mathrm{MW} / \mathrm{cm}^{2}$ gives the same value $0.1 \mathrm{~cm}^{-1}$ (or $3 \mathrm{GHz}$ ) for $\varepsilon_{v}$. We will take these orders of magnitude as typical in the following.

4.1 Perturbation of atomic Rydberg states. It is well known that atomic energy levels are shifted by irradiation with non-resonant light (references are given in section 1). The calculation of those shifts requires the computation of the dipole matrix elements between the considered level and all the others, and a numerical summation of all their contributions. The effective hamiltonian, the eigenvalues of which are the perturbed energies, gives directly the result of this infinite summation. In counterpart of this great simplification, we are limited to states $(n, l)$ which fulfill the high frequency condition :

$$
-\hbar \omega \ll \varepsilon_{n l}<0 \text {. }
$$

If we exclude the case of far UV light ( $\hbar \omega>\mathrm{Ry}$ ), only weakly bound states satisfy the condition (4.1). For these states, one can then make the Rydberg state approximation, i.e. consider the unperturbed energy levels as those of a single electron moving in a core potential $U(r)$, which is central and coulombic at long range. The unperturbed Hamiltonian is then

$$
\mathscr{H}_{\mathrm{e}}=\frac{p^{2}}{2 m}+\mathcal{V}(r)+\mathscr{H}_{\mathrm{f} . \mathrm{s}}
$$

where $\mathscr{H}_{\mathrm{f} . \mathrm{s}}$ is the fine structure hamiltonian. The perturbed energy levels then appear as those of a dressed electron moving in the same potential $U(r)$.

In fact the condition (4.1) is not sufficient to ensure that the coupling of the initial state with energy levels far from it (at a distance $\hbar \omega$ or more) has negligible effects and thus that the complete high frequency condition (i) of section (2.4) is fulfilled. Photoionization or virtual transitions to deep bound states may occur. But, in such processes the velocity of the electron undergoes a large change, which implies that the electron be close to the nucleus in order to give it the recoil necessary to the momentum conservation. We thus expect the high frequency condition to be fulfilled when the electron remains far from the nucleus, that is for states with large angular momentum $(l \gg 1)$. In the following, we essentially consider such states. We will precise later the exact validity conditions of the high frequency approximation.

4.1.1 Effective hamiltonian. - The effective hamiltonian in the presence of the light wave is deduced from (2.1). One finds :

$$
\begin{aligned}
\mathscr{H}_{\mathrm{eff}} & =\mathscr{H}_{\mathrm{e}}+\mathcal{E}_{\mathrm{v}}-\frac{\mathcal{E}_{\mathrm{v}}}{m c^{2}} \frac{p^{2}}{2 m}+\frac{\mathcal{E}_{\mathrm{v}}}{3 m \omega^{2}}\left(\frac{\mathrm{d}^{2} \mathcal{V}}{\mathrm{d} r^{2}}+\frac{2}{r} \frac{\mathrm{d} \mathcal{V}}{\mathrm{d} r}\right)+ \\
& +\frac{\mathcal{E}_{\mathrm{v}}}{3 m \omega^{2}}\left(\frac{\mathrm{d}^{2} \mathcal{V}}{\mathrm{d} r^{2}}-\frac{1}{r} \frac{\mathrm{d} \mathcal{V}}{\mathrm{d} r}\right)\left(3 \frac{(\boldsymbol{\varepsilon} \cdot \mathbf{r})\left(\varepsilon^{*} \cdot \mathbf{r}\right)}{r^{2}}-1\right) .
\end{aligned}
$$

The effect of the light beam is thus accounted for by adding four terms to the atomic hamiltonian :

i) The first term $\boldsymbol{E}_{v}$ is positive and represents the oscillatory kinetic energy of the electron in the light wave ; it appears as a mass shift of the dressed electron.

ii) The second term which is much smaller gives the corresponding correction to the electron kinetic energy.

iii) The third and the fourth terms arise from the electrostatic potential averaging by the vibrating electron (apparition of an electric form factor). We have split the corresponding corrective potential into its isotropic and anisotropic parts.

None of these terms concerns the electron spin. Corrections to the spin orbit coupling indeed exist, but are smaller than the terms considered here at least by a factor $\varepsilon_{\mathrm{nl}} / \hbar \omega$. 
4.1.2 Perturbed energy diagram. - Consider now the perturbation of the atomic energy levels brought by these four new terms in the electron hamiltonian.

The first term $\varepsilon_{v}$ shifts upwards all the Rydberg levels by the same quantity and cannot be detected on a transition between these states. Nevertheless in the cases considered here, the high frequency approximation cannot be applied also to the ground state and its energy shift is different from the one of the Rydberg states. For instance, if the perturbing light has a frequency lower than the atomic resonance line, the ground state is shifted downwards (see Fig. 3). Two-photon spectroscopy between the ground state and the Rydberg states [24,25] could be used to measure the energy shift difference. In the typical examples described above, the $0.1 \mathrm{~cm}^{-1}$ expected shift is huge compared to the two-photon line width and could be easily detected.

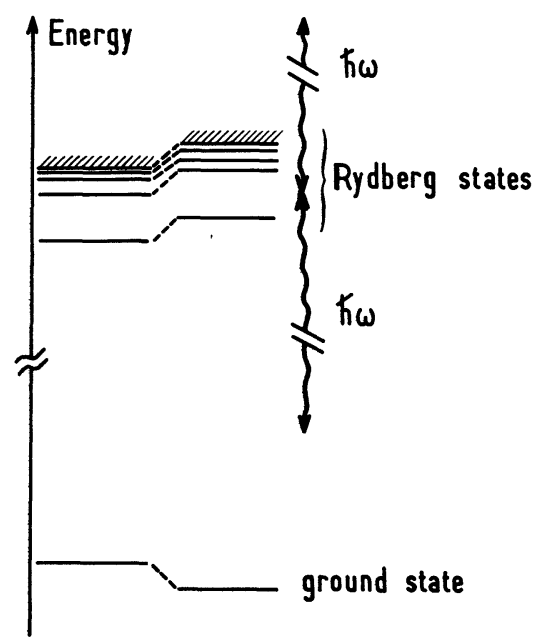

FIG. 3. - Effect of the first term of the effective hamiltonian : all the Rydberg states are shifted by the same amount with respect to the ground state which generally undergoes a different shift.

The second term changes the level energies by about $10^{-10}$ in the considered experimental examples, without perturbing the Zeeman degeneracy or the fine structure. We therefore neglect it in the following.

Only the third and fourth terms affect the relative positions of the Rydberg states. The third term commutes with the orbital angular momentum and only produces a shift of all the sublevels of a $(n, l)$ configuration by the same quantity. This shift strongly depends on the atomic pseudo-potential $V(r)$ seen by the outer electron. If $V(r)$ is simply the mean potential due to the other atomic electrons, the shift can be written as

$$
\left.\varepsilon_{\mathrm{v}} \frac{16 \pi a_{0}^{3}}{3}<-\rho(r)+Z \delta(r)\right\rangle_{n l}\left(\frac{\mathrm{Ry}}{\hbar \omega}\right)^{2}
$$

where $\rho(r)$ is the core electron density. For $l \gg 1$, the mean value of the $\delta$-function corresponding to the nucleus charge is zero, and the shift appears to be negative, proportional to the probability of finding the outer electron in the core.

The fourth term is an anisotropic zero mean value potential. For Rydberg states of many electron atoms, the fine structure is generally larger than this term and it can be treated as a perturbation in each fine structure sublevel. In each of these states, it is proportional to $\frac{3}{2}(\boldsymbol{\varepsilon J})\left(\varepsilon^{*} \mathbf{J}\right)+\frac{3}{2}\left(\boldsymbol{\varepsilon}^{*} \mathbf{J}\right)(\boldsymbol{\varepsilon} \mathbf{J})-J(J+1)$, and removes the Zeeman degeneracy of the atomic level in a way similar to the Stark effect. The splitting is about $40 \mathrm{MHz}$ for $n=10 \mathrm{D}$ state of sodium irradiated by the $\mathrm{N}_{2}$ laser.

Hydrogen is a special case, because of the properties of Coulomb potential. The third term reduces to a $\delta$-function (see formula (4.4)) and only shifts the $(n, s)$ states. The other states $(l>1)$ are unaffected. The fourth term of (4.3) has to be diagonalized within the whole set of $(n, l)$ states ( $n$ fixed, and $0<l<n-1$ ), since they are degenerate. Fortunately, selection rules on both the angular and the radial parts of the matrix elements [26] make the fourth term of the effective hamiltonian diagonal in $l$. For a $(n, l)$ level, it is equal to :

$$
\begin{gathered}
\frac{16}{n^{3}(2 l-1)(2 l+1)(2 l+3)} \varepsilon_{\mathrm{v}}\left(\frac{\mathrm{Ry}}{\hbar \omega}\right)^{2} \times \\
\times \frac{3(\boldsymbol{\varepsilon} . \mathbf{L})\left(\varepsilon^{*} . \mathbf{L}\right)-l(l+1) \hbar^{2}+\text { c.c. }}{2 l(l+1) \hbar^{2}} .
\end{gathered}
$$

The ratio of this term to the $(n, l)$ level fine structure is approximately $\varepsilon_{\mathrm{v}} m c^{2} / l(\hbar \omega)^{2}$ and can be either smaller than 1 (it splits each fine structure state in a Stark-line pattern), or larger than 1 (the fine structure is then decoupled by the perturbation into separate spin doublets).

4.1.3 Discussion. - We have considered so far that the excited electron moves in the unperturbed core potential $V(r)$. In fact, the atomic core is also subjected to the light wave and becomes polarized : this oscillating dipole interacts with the oscillating electron and creates another effective interaction potential between the core and the dressed electron. Quantum mechanically, this phenomenon is accounted for by the coupling of the one electron spectrum with the many excited electron spectra. If no particular resonance occurs, the corresponding effects are smaller than the fourth term of (4.3) by a factor $(\hbar \omega)^{2} / \mathrm{Ry} \hbar \omega_{\mathrm{e}}$ ( $\hbar \omega_{\mathrm{e}}$ being the core excitation energy).

We have already seen that the validity of the high frequency approximation seems questionable for low values of $l$. We have to discuss the magnitude of the matrix elements of $\mathcal{H}_{I_{1}}$ (formula (2.8)) between the Rydberg state $(n, l)$ and other energy levels $\mathcal{E}^{\prime}$, either in the discrete spectrum or in the continuum, which do not satisfy the condition $\left|\varepsilon_{n l}-\varepsilon^{\prime}\right| \ll \hbar \omega$, and we have to verify under what conditions their effects 
are smaller than those studied so far which are of the order of $\varepsilon_{\mathrm{v}}\left(\frac{\varepsilon_{n l}}{\hbar \omega}\right)^{2}$. The leading term of $\mathscr{H}_{\mathrm{I}_{1}}$ is $\frac{e}{m} \mathbf{A}_{\mathrm{r}} \mathbf{p}$ and its matrix elements are more easily evaluated in momentum space. We separate the angular part of the matrix element which obeys the selection rule $\Delta l= \pm 1$ and the radial part. Apart from the propagation factors in $\mathbf{A}_{\mathbf{r}}$, which account for the photon momentum and which are negligible (the photon momentum is much smaller than that of the atomic electron), the radial matrix element is diagonal in $p$. Thus, if the wave functions of two atomic states do not overlap in the momentum space, they are not coupled by the interaction with the light wave. The radial momentum wave function of the Rydberg state $(n, l)$ is centered at $p \approx \sqrt{2 m\left|\varepsilon_{n l}\right|}$ (see ref. [27]). Its extension around this mean value is weak for $l \approx n$ (classically, the electron is orbiting at a nearly constant speed) and is much broader for low $l$ (large speeds are reached by the electron near the nucleus).

Consider first the coupling of the Rydberg state with deep bound states $\left(\mathcal{E}_{n^{\prime} l^{\prime}} \lesssim-\hbar \omega\right)$. The extension of such deep bound states in the momentum space is roughly $\sqrt{2 m \hbar \omega}$ and the two wave functions clearly overlap. Hence, the radial matrix elements are generally not small. For instance, one can easily find that in hydrogen the coupling with the ground state produces a shift of the $(n, l=1)$ state of the order of $\varepsilon_{\mathrm{v}}\left(\mathcal{E}_{n l} / \hbar \omega\right)^{3 / 2}$, larger than the effects considered here. But if we restrict ourselves to Rydberg states such that $l \gg \sqrt{\mathrm{Ry} / \hbar \omega}$, they are coupled only to states $\left(n^{\prime}, l^{\prime}\right)$ such that $l^{\prime}=l \pm 1$, and hence $n^{\prime} \gg \sqrt{\mathrm{Ry} / \hbar \omega}$; the energy of such states verifies $-\hbar \omega \ll-\mathrm{Ry} / n^{\prime 2}<0$, so that all the bound states, to which the Rydberg state is coupled, fill the high frequency condition $\left|\varepsilon_{n l}-\varepsilon_{n^{\prime} l^{\prime}}\right| \ll \hbar \omega$.

The Rydberg state $(n, l)$ is also coupled to continuum states whose energy is equal to or larger than $\varepsilon_{n l}+\hbar \omega \simeq \hbar \omega$. In momentum space, the wave function of a continuum state with energy $\varepsilon$ has a very small value for $0<p<\sqrt{2 m \varepsilon}$ (the probability to find the electron with a velocity smaller than its velocity at infinity is very small). The momentum distribution is mainly centered around $\sqrt{2 m \varepsilon}$, and decreases at infinity as a power of $1 / p$ which increases with $l$. Thus for $\varepsilon \gtrsim \hbar \omega$, the continuum wave function overlaps only the large $p$ tail of the Rydberg state, for which analytical forms for hydrogenic wavefunctions are easily found [27]. Starting from those considerations, the level shift and the broadening due to the coupling with the continuum can be roughly evaluated. They appear to be smaller than the terms considered here, provided that $l \gg \sqrt{\mathrm{Ry} / \hbar \omega}$. This condition has a simple semi-classical interpretation : the maximum electron momentum in the Rydberg state is reached when the electron is at its closest distance from the nucleus and is equal to $\hbar / l a_{0}$; if it is smaller than the minimum momentum $\sqrt{2 m \hbar \omega}$ for the continuum state, the two wave functions do not overlap and matrix elements are very small. Finally for Rydberg states, the high frequency condition is fulfilled if

$$
-\hbar \omega \ll \varepsilon_{n l}<0
$$

and

$$
\sqrt{\frac{\mathbf{R y}}{\hbar \omega}} \ll l
$$

One must also mention that, for very high $n$ Rydberg states, energy shifts become smaller than the width of the levels due to Raman and Compton scattering (these processes are roughly independent of $n$ and are characterized by a cross-section of the order of $r_{0}^{2}$, where $r_{0}$ is the electron classical radius). A simple calculation shows that $n$ is thus restricted by :

$$
n<\left(\alpha^{-1} \mathrm{Ry} / \hbar \omega\right)^{3 / 4}
$$

which is about $n \approx 100$ for $\mathrm{N}_{2}$ laser light, $n \approx 1000$ for $\mathrm{CO}_{2}$ laser.

To conclude, Rydberg states are not in general dramatically affected by powerful optical irradiation. But observable changes of the level energies are nevertheless expected, which can be described by a simple effective hamiltonian. It would be interesting to investigate in more detail the case of low angular momentum states, for which the high frequency approximation made in the present theory is not appropriate.

4.2 MODIFICATION OF THE CYCLOTRON AND SPIN PRECESSION FREQUENCIES IN A STATIC MAGNETIC FIELD. - We investigate now how the cyclotron and spin precession frequencies of an electron are modified by high frequency irradiation. We consider an electron orbiting in a constant uniform magnetic field $\mathbf{B}_{0}$ with a non-relativistic energy. As explained in section (2.3), we neglect in this paper the radiative corrections to the electron $g$-factor so that the cyclotron and the spin precession frequencies are equal :

$$
\omega_{0}=\omega_{\mathrm{s}}=\frac{e B_{0}}{m}
$$

The uniform magnetic field is described by the usual vector potential :

$$
\mathbf{A}=\frac{1}{2} \mathbf{B}_{0} \times \mathbf{r}
$$

The non-relativistic limit of the unperturbed hamiltonian $\mathscr{H}_{\mathrm{e}}$ is sufficient for our purpose (the electron is orbiting with a non-relativistic energy) :

$$
\begin{aligned}
\mathscr{H}_{\mathrm{e}} & =\frac{\pi_{0}^{2}}{2 m}+\frac{-e \hbar}{2 m} \boldsymbol{\sigma} \cdot \mathbf{B}_{0} \\
\boldsymbol{\pi}_{0} & =\mathbf{p}-e \mathbf{A}_{0} .
\end{aligned}
$$


For a given value of the velocity along $\mathbf{B}_{0}$, the eigenvalues of the kinetic energy $\pi_{0}^{2} / 2 m$ are the Landau level energies $q \hbar \omega_{0}(q=0,1,2, \ldots)$; the spin magnetic energy is $\pm \frac{1}{2} \hbar \omega_{\mathrm{s}}$, so that one gets the energy diagram represented on figure $4 a$. As $\omega_{0}=\omega_{\mathrm{s}}$, there is a degeneracy between the two levels $(q,+)$ and $(q+1,-)$. Using the matrix elements of $\mathbf{r}, \boldsymbol{\pi}_{0}$ and $\boldsymbol{\sigma}$ between these energy levels, it is easy to show that the interaction of the electron with the i.e.w. only couples adjacent energy levels in the non-relativistic limit,

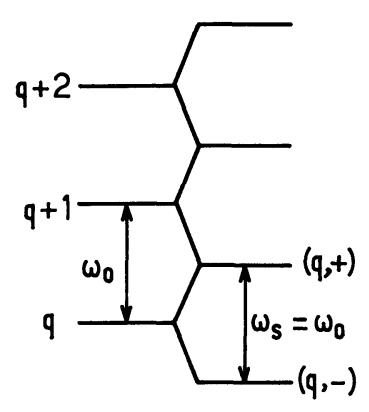

(a)

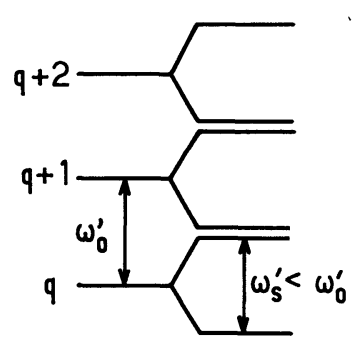

(b)
FIG. 4. - a) Unperturbed energy diagram of an electron in a magnetic field (radiative corrections to the electron $g$ factor have been neglected). b) Energy diagram in an intense electromagnetic wave.

and obeys the selection rule $\Delta q \leqslant 3$ if the $1 / c^{2}$ corrections are included. Thus if $\omega_{0}, \omega_{\mathrm{S}} \ll \omega$, the high frequency condition is very well fulfilled. The motion in presence of the i.e.w. is described by the effective hamiltonian deduced from expressions (3.1) and (3.12) :

$$
\begin{aligned}
& \mathscr{H}_{\text {eff }}=\frac{\pi_{0}^{2}}{2 m}-\frac{\varepsilon_{\mathrm{v}}}{m c^{2}} \frac{\pi_{0}^{2}}{2 m}+ \\
& +(\boldsymbol{\mu} . \boldsymbol{\kappa})\left(\frac{\mathbf{B}_{0} \cdot \pi_{0}}{m c}-2 \frac{\left(\boldsymbol{\kappa} \cdot \mathbf{B}_{0}\right)\left(\boldsymbol{\kappa} \cdot \pi_{0}\right)}{m c}\right)+ \\
& +\left(\frac{-e \hbar}{2 m}\right) \boldsymbol{\sigma} . \mathbf{B}_{0}-\frac{\varepsilon_{\mathrm{v}}}{m c^{2}}\left(\frac{-e \hbar}{2 m}\right)\left[\frac{\left(\boldsymbol{\kappa} . \mathbf{B}_{0}\right)(\boldsymbol{\kappa} . \boldsymbol{\sigma})}{2}+\right. \\
& \left.+(\boldsymbol{\varepsilon} . \boldsymbol{\sigma})\left(\boldsymbol{\varepsilon}^{*} \cdot \mathbf{B}_{0}\right)+((\boldsymbol{\kappa} \times \boldsymbol{\varepsilon}) \cdot \boldsymbol{\sigma})\left(\left(\boldsymbol{\kappa} \times \boldsymbol{\varepsilon}^{*}\right) . \mathbf{B}_{0}\right)+\text { c.c. }\right] .
\end{aligned}
$$

In this expression of $\mathcal{H}_{\mathrm{eff}}$, constant terms independent of the electron variables have been discarded; $\boldsymbol{\mu}$ is defined by formula (3.7).

The effective hamiltonian is the sum of an orbital hamiltonian (first two lines) and a spin hamiltonian (last two lines). The cyclotron motion and the spin precession remain decoupled and can be studied separately. The new terms displayed by $\mathscr{H}_{\text {eff }}$ represent the dressing of the electron by the i.e.w. and have the following consequences :

i) The cyclotron motion is slowed down. - Let us suppose first that the i.e.w. is linearly polarized; $\boldsymbol{\mu}=0$ and the term of the second line vanishes. The orbital hamiltonian reduces to $\pi_{0}^{2} / 2 m_{\text {eff }}$, taking into account the increased effective mass of the dressed electron defined by (3.2). The cyclotron frequency is proportional to $\mathrm{m}^{-1}$ so that the new cyclotron pulsation $\omega_{0}^{\prime}$ is smaller than $\omega_{0}$ :

$$
\omega_{0}^{\prime}=\omega_{0}\left(1-\frac{\varepsilon_{\mathrm{v}}}{m c^{2}}\right)
$$

For an arbitrary polarization, the third term is nonzero, but does not change the previous result. It is linear in $\pi_{0}$ and can be written as $-\mathbf{P} . \pi_{0}$, where $\mathbf{P}$ is a vector proportional to $\varepsilon_{\mathrm{v}}$. Neglecting terms in $\varepsilon_{\mathrm{v}}^{2}$, we can express the whole first line of (4.13) as $\left(\pi_{0}-\mathbf{P}\right)^{2} / 2 m_{\text {eff }}$. The components of $\mathbf{P}$ are $c$-numbers; the commutation rules of the components of $\pi_{0^{\prime}}-\mathbf{P}$ are identical to those of $\pi_{0}$ so that the hamiltonian $\left(\pi_{0}-\mathbf{P}\right)^{2} / 2 m_{\text {eff }}$ has the same Bohr frequencies than $\pi_{0}^{2} / 2 m_{\text {eff }}$. It can be shown that the only physical consequence of this third term is a very slow drift velocity $\mathbf{P} / m$ added to the cyclotron motion.

Remark : One must remember that the evolution of the expectation values of the electronic observables cannot be computed simply by putting $\mathscr{H}_{\text {eff }}$ in the Ehrenfest (or Heisenberg) equations. The observables must be transformed first by $U^{+}$as mentioned in paragraph 2.2 (remark ii).

ii) The spin precession is slowed down more than the cyclotron motion. - The second line of (4.13) may be interpreted as a Zeeman spin hamiltonian in a magnetic field $\mathbf{B}_{0}^{\prime}$ slightly different from $\mathbf{B}_{0}$. For circular or linear polarization, this apparent magnetic field $\mathbf{B}_{0}^{\prime}$ seen by the spin has a simple expression which can be easily calculated from (3.14) :

$$
\mathbf{B}_{0}^{\prime}=\mathbf{B}_{0}-\frac{\varepsilon_{\mathrm{v}}}{m c^{2}}\left(2 \mathbf{B}_{0}-\mathbf{\kappa}\left(\boldsymbol{\kappa} . \mathbf{B}_{0}\right)\right) .
$$

The component of $\mathbf{B}_{0}$ along the wave vector is reduced by $\left(1-\varepsilon_{\mathrm{v}} / m c^{2}\right)$, the other ones by $\left(1-2 \varepsilon_{\mathrm{v}} / m c^{2}\right)$. The perturbed spin precession is $\omega_{\mathrm{S}}^{\prime}=(-e \hbar / 2 m) B_{0}^{\prime}$. According to (4.15), it depends on the direction of $\mathbf{B}_{0}$, but lies clearly between two limits :

$\omega_{\mathrm{S}}\left(1-2 \frac{\varepsilon_{\mathrm{v}}}{m c^{2}}\right) \leqslant \omega_{\mathrm{S}}^{\prime} \leqslant \omega_{\mathrm{S}}\left(1-\frac{\varepsilon_{\mathrm{v}}}{m c^{2}}\right)$.

It can be easily shown that this result holds also for all the possible polarizations of the i.e.w. Therefore the spin precession frequency is always reduced by the interaction with the i.e.w. A more careful investigation of the contribution of the 4 different physical processes described in section (3.2) shows that : (i) The first two processes (smearing of the spin magnetic moment over a finite angle and increase of the electron mass) always reduce $\omega_{\mathbf{s}}$; (ii) The corrections associated with the last two processes (motional 
fields and rectification effects) may have both signs, but cannot change the sign of the overall correction which is always negative. Comparing (4.16) to (4.14), it appears that the spin precession frequency is reduced more than the cyclotron frequency. It follows that the energy diagram of figure $4 a$ is perturbed as shown in figure $4 b$. To realize experimentally such an energy diagram, stimulated corrections are required to be at least $10^{-2}$ (at least larger than the spontaneous radiative corrections which are known to be $10^{-3}$ and that we have neglected so far). To achieve this, one finds that a light flux of about $10^{15} \mathrm{~W} / \mathrm{cm}^{2}$ would be necessary. Such a light intensity is for the moment beyond the experimental possibilities. With the available fluxes given in the introduction of section 4 , the corrections to the cyclotron and spin precession frequencies are expected to be of the order of $10^{-9}$.

The results discussed in this section are nevertheless interesting from a theoretical point of view, in connection with the questions raised in the introduction. Many attempts have been made to derive simply the $g-2$ correction of the electron magnetic moment $[28,20]$. Following Welton [28] some of these derivations try to interpret $g-2$ as resulting from the vibration of the electron spin induced by the electromagnetic field vacuum fluctuations, considered as an applied field. From the results obtained in the present paper, we think that we can put forward the following points :

(i) A consistent calculation must start from an electronic hamiltonian which includes relativistic corrections to the interaction between the electron and the electromagnetic field (mass correction, spinorbit coupling, retardation effects). Taking into account only the coupling of the spin magnetic moment with the magnetic field of the i.e.w. leads to incomplete results.

(ii) Even a consistent calculation, which includes relativistic corrections, fails to produce an enhancement of the electron magnetic moment. If one averages over the polarization and the direction of the wave, the electron $g$-factor is in fact more reduced than a neutral particle $g$-factor. This fact can be clearly attributed to the existence of the electron charge, which causes supplementary couplings between the spin and the fields. Thus, one cannot invoke the electron charge nor the relativistic nature of the spin to explain the failure of a Welton type calculation.

(iii) Finally, even if one considers that only the relative magnitude of the spin precession frequency to the cyclotron frequency has a physical significance, one always finds a negative correction. This seems to indicate that spontaneous renormalization of the electron properties is qualitatively different from the stimulated one. A term to term comparison between spontaneous and stimulated effects associated to a given mode of the e.m. field would be interesting to understand this difference. The effective hamiltonian formalism developed here appears to be very well suited for such a comparison, since stimulated and spontaneous terms are given in the same way by a unique calculation (see formula (2.16)). We will consider such a problem elsewhere.

Appendix A : Calculation of the effective Hamiltonian matrix elements. - Let us rename $|a\rangle,\left|a^{\prime}\right\rangle \ldots$ the eigenstates of the unperturbed Hamiltonian $\mathcal{H}_{\mathrm{e}}+\mathcal{H}_{\mathrm{f}}$ belonging to the multiplicity $\varepsilon_{N},|b\rangle,\left|b^{\prime}\right\rangle \cdots$ those belonging to all other multiplicities $\varepsilon_{N^{\prime}}\left(N^{\prime} \neq N\right)$, and $\lambda \mathcal{H}_{\mathrm{I}}$ the perturbation which couples the states $|a\rangle$ and $|b\rangle$.

We want to find a unitary transformation $U=\mathrm{e}^{i S}$ ( $S:$ hermitian operator) which eliminates up to $\lambda^{2}$, the off-diagonal part of the coupling i.e. : For each $|a\rangle$ and $|b\rangle$ :

$$
\left\langle a\left|\mathrm{e}^{i S}\left(\mathcal{H}_{\mathrm{e}}+\mathcal{H}_{\mathrm{f}}+\lambda \mathcal{H}_{\mathrm{f}}\right) \mathrm{e}^{-i S}\right| b\right\rangle=\mathcal{O}\left(\lambda^{2}\right) .
$$

As a matter of fact, this condition does not completely specify the transformation $\mathrm{e}^{i S}$ : if a given operator $\mathrm{e}^{i \mathrm{~S}}$ satisfies (A.1), the whole set of operators of the form $A \mathrm{e}^{i S}$ also satisfies (2.1), if $A$ is a diagonal unitary operator (i.e. only acting inside each multiplicity $\varepsilon_{N}$ ). We therefore require $S$ to be completely off-diagonal, more precisely : For each $|a\rangle,\left|a^{\prime}\right\rangle,|b\rangle,\left|b^{\prime}\right\rangle$ :

$$
\left\langle a|S| a^{\prime}\right\rangle=\left\langle b|S| b^{\prime}\right\rangle=0 \text {. }
$$

Let us now expand $S$ in powers of $\lambda$ :

$$
S=S_{0}+\lambda S_{1}+\lambda^{2} S_{2}+\cdots
$$

If we use the identity :

$$
\mathrm{e}^{i S} A \mathrm{e}^{-i S}=A+i[S, A]-\frac{1}{2}[S,[S, A]]+\cdots
$$

we are now able to expand the left side of eq. (A.1) in powers of $\lambda$. The $(\lambda)^{0}$ coefficient is 0 if we take $S_{0}=0$. Equating the $(\lambda)^{1}$ coefficient to 0 , we obtain the following equation for $S_{1}$ :

$\left\langle a\left|\mathscr{H}_{\mathrm{I}}\right| b\right\rangle+i\left\langle a\left|\left[S_{1}, \mathscr{H}_{\mathrm{e}}+\mathscr{H}_{\mathrm{f}}\right]\right| b\right\rangle=0$

which gives for the off-diagonal matrix elements of $S_{1}$ :

$$
\left\langle a\left|S_{1}\right| b\right\rangle=i \frac{\left\langle a\left|\mathcal{H}_{1}\right| b\right\rangle}{\varepsilon_{b}-\varepsilon_{a}}
$$

(all other matrix elements of $S_{1}$ are 0 ).

We can now use expression (A.6) to calculate the matrix elements of $\mathscr{H}_{\text {eff }}$ inside the multiplicity $\varepsilon_{N}$ :

$$
\begin{aligned}
& \left\langle a\left|\mathcal{H}_{\text {eff }}\right| a^{\prime}\right\rangle=\left\langle a\left|\mathrm{e}^{i S}\left(\mathscr{H}_{\mathrm{e}}+\mathcal{H}_{\mathrm{f}}+\lambda \mathcal{H}_{\mathrm{I}}\right) \mathrm{e}^{-i S}\right| a^{\prime}\right\rangle= \\
& =\left\langle a\left|\mathscr{H}_{\mathrm{e}}+\mathscr{H}_{\mathrm{f}}\right| a^{\prime}\right\rangle+i \lambda^{2}\left\langle a\left|\left[S_{1}, \mathscr{H}_{\mathrm{l}}\right]\right| a^{\prime}\right\rangle- \\
& -\frac{1}{2} \lambda^{2}\left\langle a\left|\left[S_{1},\left[S_{1}, \mathscr{H}_{0}+\mathscr{H}_{\mathrm{e}}\right]\right]\right| a^{\prime}\right\rangle+\cdots
\end{aligned}
$$

We have used eq. (A.4) and condition (A.2). $S_{2}$ only contributes to higher order terms in $\lambda$. So, we only need the expression (A.6) of $S_{1}$ in order to 
compute $\mathscr{H}_{\text {eff }}$ up to second order in $\lambda$. After a few calculations, we obtain the final expression of the matrix elements of $\mathscr{H}_{\text {eff }}$ inside the multiplicity $\mathcal{E}_{N}$ :

$$
\begin{aligned}
&\left\langle a\left|\mathscr{H}_{\text {eff }}\right| a^{\prime}\right\rangle=\left\langle a\left|\mathscr{H}_{\mathrm{e}}+\mathscr{H}_{\mathrm{f}}\right| a^{\prime}\right\rangle+ \\
&+\frac{1}{2} \lambda^{2} \sum_{b}\left(\frac{1}{\mathcal{E}_{a}-\varepsilon_{b}}+\frac{1}{\mathcal{E}_{a^{\prime}}-\varepsilon_{b}}\right) \times \\
& \times\left\langle a\left|\mathscr{H}_{\mathrm{I}}\right| b\right\rangle\left\langle b\left|\mathcal{H}_{\mathrm{I}}\right| a^{\prime}\right\rangle .
\end{aligned}
$$

If we come back to the notations of paragraph 2.2, we are led to the expression of eq. (2.12).

Appendix B : Classical vibration of an electron in a plane electromagnetic wave. - ELECTRIC AND MAGNETIC FIELDS OF THE PLANE WAVE. - The expressions of $\mathbf{E}_{\mathbf{r}}(\mathbf{r}, t)$ and $\mathbf{B}_{\mathbf{r}}(\mathbf{r}, t)$ are obtained by replacing the corresponding quantum operators $a$ and $a^{+}$by $\sqrt{N} \mathrm{e}^{-i \omega t}$ and $\sqrt{N} \mathrm{e}^{i \omega t}$

$$
\begin{aligned}
& \mathbf{E}_{\mathbf{r}}(\mathbf{r}, t)=\frac{i E}{\sqrt{2}}\left(\mathrm{e}^{i \mathbf{k} . \mathbf{r}} \mathrm{e}^{-i \omega t} \varepsilon-\mathrm{e}^{-i \mathbf{k} \cdot \mathbf{r}} \mathrm{e}^{i \omega t} \varepsilon^{*}\right) \\
& \mathbf{B}_{\mathbf{r}}(\mathbf{r}, t)=\frac{i E}{c \sqrt{2}}\left(\mathrm{e}^{i \mathbf{k} \cdot \mathbf{r}} \mathrm{e}^{-i \omega t} \mathbf{\kappa} \times \varepsilon-\mathrm{e}^{-i \mathbf{k} \cdot \mathbf{r}} \mathrm{e}^{i \omega t} \varepsilon^{*}\right)
\end{aligned}
$$

- Vibration of the Electron. - Since we are interested in small corrections due to the vibration of the electron, one can show that it is sufficient to calculate this vibration to zeroth order in $v / c$. So, we will neglect the effect of the magnetic force $e \mathbf{v} \times \mathbf{B}_{\mathrm{r}}$, which is $v / c$ times smaller than the electric force $e \mathbf{E}_{\mathrm{r}}$, and we will replace $\mathrm{e}^{ \pm i \mathbf{k r}}$ by 1 , which gives for $\mathbf{E}_{\mathrm{r}}$ :

$$
\mathbf{E}_{\mathrm{r}}(t)=\frac{i E}{\sqrt{2}}\left(\mathrm{e}^{-i \omega t} \varepsilon-\mathrm{e}^{i \omega t} \varepsilon^{*}\right) .
$$

The equation of motion of the electron is :

$m \frac{\mathrm{d} \mathbf{v}}{\mathrm{d} t}=\frac{i e E}{\sqrt{2}}\left(\mathrm{e}^{-i \omega t} \boldsymbol{\varepsilon}-\mathrm{e}^{i \omega t} \varepsilon^{*}\right)+e \mathbf{E}_{0}+e \mathbf{v} \times \mathbf{B}_{0}$

where $\mathbf{E}_{0}$ and $\mathbf{B}_{0}$ are the static electric and magnetic fields which are eventually applied.

(i) Vibration in absence of external static fields $\left(\mathbf{E}_{0}=\mathbf{B}_{0}=0\right)$. - Let us call $\rho$ the deviation of the electron from its average position. When $\mathbf{E}_{0}=\mathbf{B}_{0}=0$, eq. (B.4) is readily integrated to give :

$$
\mathbf{v}=-\frac{e E}{m \omega \sqrt{2}}\left(\mathrm{e}^{-i \omega t} \varepsilon+\mathrm{e}^{i \omega t} \varepsilon^{*}\right)
$$

$\boldsymbol{\rho}=-i \frac{e E}{m \omega^{2} \sqrt{2}}\left(\mathrm{e}^{-i \omega t} \varepsilon-\mathrm{e}^{i \omega t} \varepsilon^{*}\right)=-\frac{e}{m \omega} \mathbf{E}_{\mathrm{r}}(t)$
The electron vibrates in phase with $\mathbf{E}_{\mathrm{r}}(e<0)$.

"For a linear polarization $\left(\varepsilon=\varepsilon^{*}\right)$, the electron vibrates along $\varepsilon$ with an amplitude $e E \sqrt{2} / m \omega^{2}$ (Fig. 5a).

For a circular polarization, it moves at frequency $\omega$ on a small círcle of radius $e E / m \omega^{2}$, in a plane perpendicular to к (Fig. 5b).

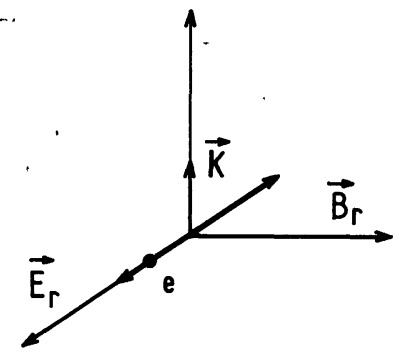

a)

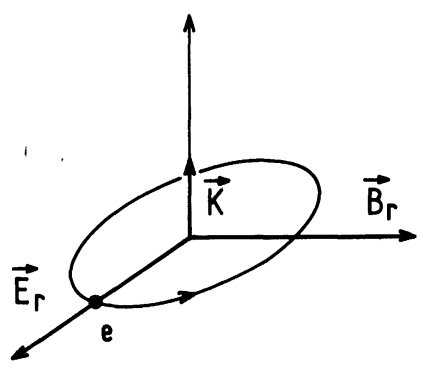

b)

FIG. 5. - Classical motion of an electron : $a$ ) in a linearly polarized plane wave, $b)$ in a circularly polarized plane wave $\left(\mathbf{E}_{\mathrm{r}}, \mathbf{B}_{\mathrm{r}}\right.$ electric and magnetic fields of the plane wave).

In all cases, the mean kinetic energy (averaged over a period $2 \pi / \omega$ of the i.e.w.) is :

$\varepsilon_{\mathrm{v}}=\overline{\frac{1}{2} m v^{2}}=\frac{1}{4} \frac{e^{2} E^{2}}{m \omega^{2}}\left(\varepsilon \cdot \varepsilon^{*}+\varepsilon^{*} \cdot \varepsilon\right)=\frac{e^{2} E^{2}}{2 m \omega^{2}}$.

(ii) Vibration in presence of external static fields. The electric force $e \mathbf{E}_{0}$ is static and cannot affect the high frequency vibration of the electron. This is not the case for the magnetic force $e \mathbf{v} \times \mathbf{B}_{0}$ which oscillates at frequency $\omega$ as a consequence of the modulation of $\mathbf{v}$. So, we will completely ignore $\mathbf{E}_{\mathbf{0}}$ in the following.

We are interested in corrections to the electronic vibration to first order in $B_{0}$. So, we can replace in the magnetic force $e v \times \mathbf{B}_{0}$ of eq. (B.4) $\mathbf{v}$ by the zeroth order solution (B.5), independent of $\mathbf{B}_{0}$. The equation of motion so obtained is readily integrated to give :

$$
\begin{array}{r}
\mathbf{v}=-\frac{e E}{m \omega \sqrt{2}}\left(\mathrm{e}^{-i \omega t} \varepsilon+\mathrm{e}^{i \omega t} \varepsilon^{*}\right)-i \frac{e^{2} E}{m^{2} \omega^{2} \sqrt{2}} \times \\
\quad \times\left(\mathrm{e}^{-i \omega t} \varepsilon \times \mathbf{B}_{0}-\mathrm{e}^{i \omega t} \varepsilon^{*} \times \mathbf{B}_{0}\right) \cdot(\mathrm{B} .8) \\
\mathbf{\rho}=-i \frac{e E}{m \omega^{2} \sqrt{2}}\left(\mathrm{e}^{-i \omega t} \varepsilon-\mathrm{e}^{i \omega t} \varepsilon^{*}\right)+\frac{e^{2} E}{m^{2} \omega^{3} \sqrt{2}} \times \\
\times\left(\mathrm{e}^{-i \omega t} \varepsilon \times \mathbf{B}_{0}+\mathrm{e}^{i \omega t} \varepsilon^{*} \times \mathbf{B}_{0}\right) \cdot(\mathrm{B} .9)
\end{array}
$$


The last terms of eq. (B. 8) and (B.9) give the modification introduced by $\mathbf{B}_{0}$.

For a linear polarization $\left(\varepsilon=\varepsilon^{*}\right)$, the electronic vibration which was rectilinear in the absence of $\mathbf{B}_{0}$ becomes elliptical. The large axis of the ellipse is along $\varepsilon$ and has a length $e E \sqrt{2} / m \omega^{2}$. The small axis is along $\varepsilon \times \mathbf{B}_{0}$ with a length $\frac{e E \sqrt{2}}{m \omega^{2}} \frac{e B_{0}}{m \omega}$. As $\varepsilon \times \mathbf{B}_{0}$ is not in general perpendicular to $\kappa$, the vibration of the electron gets a small component along $\boldsymbol{r}$.

For circular polarization, one can show from (B.9) that the motion of the electron consists of a circular motion at frequency $\omega$ in the plane perpendicular to $k$ with a radius

$$
\left(1-\frac{e}{m \omega} \mathbf{B}_{0} \cdot \mathbf{k}\right) \frac{e E}{m \omega^{2}},
$$

and of a vibration along $\boldsymbol{x}$ which depends on the projection of $\mathbf{B}_{0}$ in the plane perpendicular to $\boldsymbol{K}$. So, the effect of $\mathbf{B}_{\mathbf{0}}$ is to change slightly the radius of the circle of figure $5 a$ and to introduce a component of the vibration along $\boldsymbol{~}$.

Appendix C : Fictitious d.c. magnetic field associated with a circularly polarized plane wave. - We have represented on figure 6 , the electric and magnetic fields $\mathbf{E}_{\mathrm{r}}$ and $\mathbf{B}_{\mathrm{r}}$ of the circularly polarized plane wave. Let us consider the reference frame $\Sigma_{\mathrm{r}}$ rotating with $\mathbf{B}_{\mathrm{r}}$ around $\mathbf{k}$ (we suppose a right circular polarization).

In $\Sigma_{\mathrm{r}}, \mathbf{B}_{\mathrm{r}}$ is static, but the spin sees another static field $-\frac{\omega}{\gamma} \mathbf{\kappa}$ (where $\gamma=\frac{e}{m}$ is the gyromagnetic ratio), much larger than $\mathbf{B}_{\mathbf{r}}$ (Larmor's theorem). The Larmor frequency around the total field $\mathbf{B}_{\mathrm{e}}=\mathbf{B}_{\mathrm{r}}-\frac{\omega}{\gamma} \mathbf{k}$ is :

$\gamma\left(B_{\mathrm{r}}^{2}+\frac{\omega^{2}}{\gamma^{2}}\right)^{1 / 2}=\omega\left(1+\frac{e^{2} B_{\mathrm{r}}^{2}}{m^{2} \omega^{2}}\right)^{1 / 2} \approx \omega+\frac{e^{2} B_{\mathrm{r}}^{2}}{2 m^{2} \omega}$.

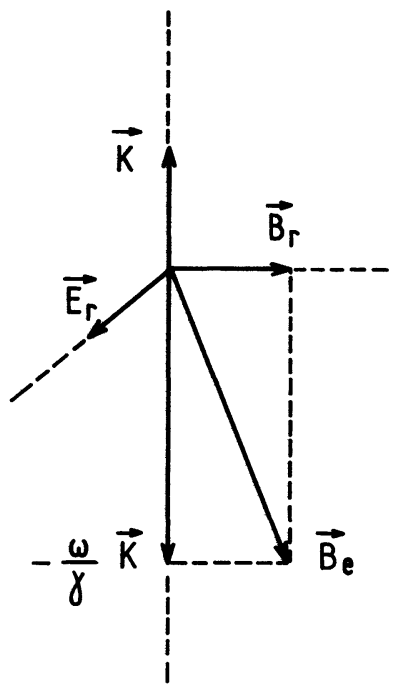

Fig. 6. - Various fields acting on the spin in the reference frame $\Sigma_{\mathrm{r}}$ rotating with $\mathbf{B}_{\mathrm{r}}$ around $\boldsymbol{\kappa}$.

When coming back to the laboratory frame, we see that the main motion of the spin is a precession around $\kappa$ with a frequency

$$
\omega-\left(\omega+\frac{e^{2} B_{\mathrm{r}}^{2}}{2 m^{2} \omega}\right)=-\frac{e^{2} B_{\mathrm{r}}^{2}}{2 m^{2} \omega} .
$$

This precession can be considered as due to a fictitious magnetic field

$$
\mathbf{B}_{\mathrm{f}}=\frac{e}{2 m} \frac{B_{\mathrm{r}}^{2}}{\omega} \mathbf{K}
$$

giving rise to a magnetic energy $-\frac{\hbar}{2 m} \sigma . \mathbf{B}_{\mathrm{f}}$ which exactly coincides with $(3.15)$ when we use the relation

$$
\left|\mathbf{B}_{\mathbf{r}}\right|=\frac{\left|\mathbf{E}_{\mathrm{r}}\right|}{c}=\frac{E}{c}
$$

between the magnetic and electric fields of a circularly polarized plane wave.

\section{References}

[1] Barrat, J. P., Cohen-Tannoudi, C., J. Phys. Radium 22 (1961) 329 and 443.

[2] Cohen-Tannoudj, C., Annls. Phys. 7 (1962) 423 and 469.

[3] Cohen-Tannoudi, C., Dupont-Roc, J., Phys. Rev. A 5 (1972) 968.

[4] HAPPER, W., Rev. Mod. Phys. 44 (1972) 169.

[5] BJorkholm, J. E., Liao, P. F., Phys. Rev. Lett. 34 (1975) 1.

[6] Aleksandrov, E. B., Bonch-Bruevich, A. M.,'Kostin, N. N., KHodovol, V. A., Sov. Phys. JETP 29 (1969) 82.

[7] Platz, P., Appl. Phys. Lett. 16 (1970) 70.

[8] Dubreuil, B., Ranson, P., Chapelle, J., Phys. Lett. 42A (1972) 323.

[9] Cohen-Tannoudj, C., Cargèse Lectures in Physics, Edited by M. Levy, Vol. 2 (Gordon and Breach) 1968.

[10] Haroche, S., Cohen-Tannoudji, C., Audouin, C., ScherMANN, J. P., Phys. Rev. Lett. 24 (1970) 861.

[11] Haroche, S., Annls. Phys. 6 (1971) 189 and 327 and refs in •
[12] SChWinger, J. S., Phys. Rev. 73 (1947) 416.

[13] Eberly, J. H., Progress in Optics VII, E. Wolf editor (North Holland) 1969 , p. 361.

[14] Landau, L. D., Lifchitz, E. M., Vol. 4, Part I : Relativistic Quantum Theory (Pergamon Press) 1971.

[15] Reiss, H. R., Eberly, J. H., Phys. Rev. 151 (1966) 1058.

[16] Berson, I., Sov. Phys. JETP 29 (1969) 871.

[17] Bagrov, V. G., Bosrikov, P. V., Gitman, D. M., Izv. Vyssh. Uchebn. Zaved. Radiofiz. 16 (1973) 129.

[18] Fedorov, M. V., KaZaKov, A. E., Z. Phys. 261 (1973) 191.

[19] JANNER, A., JansSen, T., Physica 60 (1972) 292.

[20] Lai, S. A., Knight, P. L., Eberly, J. H., Phys. Rev. Lett. 32 (1974) 494 and ref. in $\bullet$

See for instance :

Arunasalam, V., Phys. Rev. Lett. 28 (1972) 1499;

SenItZKy, I. R., Phys. Rev. Lett. 31 (1973) 955 ;

BoURRET, R., Lett. Nuovo Cimento 7 (1973) 801 ; 
ItZYKson, C., Commun. Math. Phys. 36 (1974) 19;

LAI, S. B., KNight, P. L., Eberly, J. H., Phys. Rev. Lett. 32 (1974) 494 and 35 (1975) 124 ;

BABIKER, M., Phys. Rev. A 12 (1975) 1911.

[21] Fabre, C., Thèse de $3^{\mathrm{e}}$ cycle, Paris (1974).

[22] Hegstrom, A., Phys. Rev. A 7 (1973) 451.

[23] Le Dourneuf, M., Cohen-Tannoudj, C., Dupont-Roc, J., HAROCHE, S., C. R. Hebd. Séan. Acad. Sci. 272 (1971) 1048.

[24] Levenson, M. D., Harper, C. D., Eisley, G. L., in Laser
Spectroscopy Proceedings of the 2nd International Conference, Haroche S. et al. editors (Springer Verlag) (1975) p. 452.

[25] Kato, Y., Stolcheff, B. B., J. Opt. Soc. Am. 66 (1976) 490.

[26] Pasternak, S., Sternheimer, R. M., J. Math. Phys. 3 (1962) 1280.

[27] Bethe, H. A., SAlPETER, E. E., Quantum Mechanics of oneand two-electron atoms (Springer Verlag) 1957.

[28] Welton, T. A., Phys. Rev. 74 (1948) 1157. 